\title{
Analytical approximations for matter effects on CP violation in the accelerator-based neutrino oscillations with $E \lesssim 1 \mathrm{GeV}$
}

\author{
Zhi-zhong Xing ${ }^{a, b, c}$ and Jing-yu Zhu ${ }^{a}$ \\ ${ }^{a}$ Institute of High Energy Physics, Chinese Academy of Sciences, \\ Beijing 100049, China \\ ${ }^{b}$ School of Physical Sciences, University of Chinese Academy of Sciences, \\ Beijing 100049, China \\ ${ }^{c}$ Center for High Energy Physics, Peking University, \\ Beijing 100080, China \\ E-mail: xingzz@ihep.ac.cn, zhujingyu@ihep.ac.cn
}

ABStract: Given an accelerator-based neutrino experiment with the beam energy $E \lesssim$ $1 \mathrm{GeV}$, we expand the probabilities of $\nu_{\mu} \rightarrow \nu_{e}$ and $\bar{\nu}_{\mu} \rightarrow \bar{\nu}_{e}$ oscillations in matter in terms of two small quantities $\Delta_{21} / \Delta_{31}$ and $A / \Delta_{31}$, where $\Delta_{21} \equiv m_{2}^{2}-m_{1}^{2}$ and $\Delta_{31} \equiv m_{3}^{2}-m_{1}^{2}$ are the neutrino mass-squared differences, and $A$ measures the strength of terrestrial matter effects. Our analytical approximations are numerically more accurate than those made by Freund in this energy region, and thus they are particularly applicable for the study of leptonic $\mathrm{CP}$ violation in the low-energy MOMENT, ESS $\nu \mathrm{SM}$ and T2K oscillation experiments. As a by-product, the new analytical approximations help us to easily understand why the matter-corrected Jarlskog parameter $\tilde{\mathcal{J}}$ peaks at the resonance energy $E_{*} \simeq 0.14 \mathrm{GeV}$ (or $0.12 \mathrm{GeV}$ ) for the normal (or inverted) neutrino mass hierarchy, and how the three Dirac unitarity triangles are deformed due to the terrestrial matter contamination. We also affirm that a medium-baseline neutrino oscillation experiment with the beam energy $E$ lying in the $E_{*} \lesssim E \lesssim 2 E_{*}$ range is capable of exploring leptonic CP violation with little matter-induced suppression.

KEYWORDS: CP violation, Neutrino Physics

ARXIV EPRINT: 1603.02002 


\section{Contents}

1 Introduction 1

2 The matter-enhanced Jarlskog parameter 4

3 The matter-deformed unitarity triangles 12

4 Neutrino oscillations and CP violation $\quad 21$

$\begin{array}{lll}5 & \text { Summary } & 31\end{array}$

\section{Introduction}

In the past two decades we have witnessed a booming period in neutrino physics thanks to a number of indisputable observations of atmospheric, solar, reactor and accelerator neutrino oscillations [1], and thus achieved a smoking gun for the incompleteness of the standard model (SM) in particle physics - the neutrinos actually have finite rest masses and the lepton flavors are significantly mixed, motivating us to explore the other unknowns of massive neutrinos beyond the SM and search for their possible consequences in nuclear physics, particle astrophysics and cosmology.

In the standard three-flavor scheme there are six neutrino oscillation parameters: two independent neutrino mass-squared differences (e.g., $\Delta_{21} \equiv m_{2}^{2}-m_{1}^{2}$ and $\Delta_{31} \equiv m_{3}^{2}-$ $m_{1}^{2}$ ), three lepton flavor mixing angles (i.e., $\theta_{12}, \theta_{13}$ and $\theta_{23}$ ) and one CP-violating phase (i.e., $\delta$ ). Among them, the sign of $\Delta_{31}$ and the size of $\delta$ remain unknown [2-4]. But some preliminary hints for $\delta \sim 3 \pi / 2$ and $\Delta_{31}>0$ have recently been seen by combining the $\mathrm{T} 2 \mathrm{~K}[5,6]$ and $\mathrm{NO} \nu \mathrm{A}[7]$ data on $\nu_{\mu} \rightarrow \nu_{e}$ oscillations with the Daya Bay (reactor $\bar{\nu}_{e} \rightarrow \bar{\nu}_{e}$ oscillation [8,9]) and Super-Kamiokande (atmospheric $\nu_{\mu} \rightarrow \nu_{\mu}$ oscillation [10]) data [11]. Provided $\delta$ is really around $3 \pi / 2$ or takes a nontrivial value far away from 0 and $\pi$, then remarkable $\mathrm{CP}$ - and $\mathrm{T}$-violating effects will emerge in some upcoming long-baseline neutrino oscillation experiments.

Among a number of ongoing and proposed accelerator-based experiments which aim to probe or constrain CP violation in neutrino oscillations [12], those with the beam energy $E \lesssim 1 \mathrm{GeV}$ (e.g., T2K [5], MOMENT [13] and $\operatorname{ESS} \nu \mathrm{SM}$ [14]) are expected to involve much smaller terrestrial matter effects. To understand the salient features of the matter-corrected $\nu_{\mu} \rightarrow \nu_{e}$ and $\bar{\nu}_{\mu} \rightarrow \bar{\nu}_{e}$ oscillations in this energy region, it is important to expand their probabilities in terms of two small expansion parameters $\alpha \equiv \Delta_{21} / \Delta_{31}$ and $\beta \equiv A / \Delta_{31}$, where $A \equiv 2 \sqrt{2} G_{\mathrm{F}} N_{e} E$ with $G_{\mathrm{F}}$ being the Fermi constant and $N_{e}$ being the background density of electrons. But the previous analytical approximations in this connection, such as the popular one developed by Freund [15], are usually subject to $E \gtrsim 0.5 \mathrm{GeV}$ and will 
become invalid when $E$ approaches vanishing. ${ }^{1}$ The reason is simply that mainly the longbaseline neutrino oscillation experiments with $E \gtrsim 1 \mathrm{GeV}$ were considered in those works.

Hence our present work is well motivated to offer the hitherto most systematic and useful analytical approximations for terrestrial matter effects on $\mathrm{CP}$ violation in the mediumbaseline neutrino oscillation experiments with the beam energy $E \lesssim 1 \mathrm{GeV}$.

The strength of $\mathrm{CP}$ and $\mathrm{T}$ violation in neutrino oscillations is measured by a universal and rephasing-invariant quantity of the $3 \times 3$ Pontecorvo-Maki-Nakagawa-Sakata (PMNS) lepton flavor mixing matrix $U$ [17-19], the so-called Jarlskog parameter $\mathcal{J}$ [20] defined via

$$
\operatorname{Im}\left(U_{\alpha i} U_{\beta j} U_{\alpha j}^{*} U_{\beta i}^{*}\right)=\mathcal{J} \sum_{\gamma} \epsilon_{\alpha \beta \gamma} \sum_{k} \epsilon_{i j k},
$$

where the Greek and Latin subscripts run over $(e, \mu, \tau)$ and $(1,2,3)$, respectively. When a neutrino beam travels through a medium, it can see two kinds of refractive indices because of its interactions with the constituents of the medium (i.e., electrons, protons and neutrons) via the weak neutral current (NC) and charged current (CC) [21, 22]. All the three neutrino flavors share a common "matter" phase due to the refractive index arising from the NC forward scattering, but the electron neutrinos develop an extra "matter" phase owing to the CC forward scattering. The latter is nontrivial, and hence it is likely to change the neutrino oscillation behavior. In this case one may define the matter-corrected neutrino masses $\widetilde{m}_{i}$ and the corresponding PMNS matrix $\widetilde{U}$, so as to express the probabilities of neutrino oscillations in matter in the same way as those in vacuum. For example, the T-violating asymmetry between the probabilities of $\nu_{\mu} \rightarrow \nu_{e}$ and $\nu_{e} \rightarrow \nu_{\mu}$ oscillations in matter is given by $[23,24]^{2}$

$$
\widetilde{\mathcal{A}}_{\mathrm{T}}=-16 \widetilde{\mathcal{J}} \sin \frac{\widetilde{\Delta}_{21} L}{4 E} \sin \frac{\widetilde{\Delta}_{31} L}{4 E} \sin \frac{\widetilde{\Delta}_{32} L}{4 E},
$$

in which $E$ denotes the neutrino beam energy, $L$ is the distance between a neutrino source and the detector, $\widetilde{\mathcal{J}}$ and $\widetilde{\Delta}_{i j}$ are the matter-corrected counterparts of $\mathcal{J}$ and $\Delta_{i j}$ (for $i j=21,31,32)$, respectively. It is known that $\widetilde{\mathcal{J}} \widetilde{\Delta}_{21} \widetilde{\Delta}_{31} \widetilde{\Delta}_{32}=\mathcal{J} \Delta_{21} \Delta_{31} \Delta_{32}$ exactly holds for a constant matter profile [25-27]. But a more transparent relationship between $\widetilde{\mathcal{J}}$ and $\mathcal{J}$, which can directly tell us why or how $\mathrm{CP}$ violation in matter is enhanced or suppressed as compared with that in vacuum, has been lacking. It should be noted that $\mathcal{J}$ (or $\widetilde{\mathcal{J}}$ ) is in principle a measurable quantity, but in practice it is not directly observable since it is always correlated with the oscillation terms as shown in eq. (1.2).

However, a careful study of the ratio $\widetilde{\mathcal{J}} / \mathcal{J}$ changing with the neutrino (or antineutrino) beam energy $E$ is not only conceptually interesting but also practically indispensable for expanding the matter-corrected oscillation probabilities $\widetilde{P}\left(\nu_{\mu} \rightarrow \nu_{e}\right)$ and $\widetilde{P}\left(\bar{\nu}_{\mu} \rightarrow \bar{\nu}_{e}\right)$ in

\footnotetext{
${ }^{1} \mathrm{Xu}$ has noticed that the approximate formulas obtained by Freund [15] are still valid even near the solar neutrino resonance in matter (i.e., $A \simeq \Delta_{21} \cos 2 \theta_{12}$ ) [16], but we are going to show that they will become problematic in the $E \lesssim 0.4 \mathrm{GeV}$ region and definitely turn to be invalid in the $E \lesssim 0.1 \mathrm{GeV}$ region.

${ }^{2}$ Since an ordinary medium (e.g., the Earth) only consists of electrons, protons and neutrons instead of both these particles and their antiparticles, the matter background is not symmetric under the CP transformation. Hence the expression of the CP-violating asymmetry between $P\left(\nu_{\mu} \rightarrow \nu_{e}\right)$ and $P\left(\bar{\nu}_{\mu} \rightarrow \bar{\nu}_{e}\right)$ is not so simple as that of $\widetilde{\mathcal{A}}_{\mathrm{T}}$ in eq. (1.2), as one can clearly see in section 4 .
} 
terms of the afore-defined small parameters $\alpha$ and $\beta$ in the $E \lesssim 1 \mathrm{GeV}$ region. So we plan to organize the remaining parts of this paper in an easy-to-follow and step-by-step way: starting from the analytical approximation of $\widetilde{\mathcal{J}} / \mathcal{J}$, passing through those of $\left|\widetilde{U}_{e i} \widetilde{U}_{\mu i}^{*}\right|$, $\left|\widetilde{U}_{\mu i} \widetilde{U}_{\tau i}^{*}\right|$ and $\left|\widetilde{U}_{\tau i} \widetilde{U}_{e i}^{*}\right|$ (for $\left.i=1,2,3\right)$, and ending with those of $\widetilde{P}\left(\nu_{\mu} \rightarrow \nu_{e}\right)$ and $\widetilde{P}\left(\bar{\nu}_{\mu} \rightarrow \bar{\nu}_{e}\right)$.

In section 2 we aim to reveal a unique range of the neutrino beam energy $E$ in which the size of the effective Jarlskog invariant $\widetilde{\mathcal{J}}$ can be enhanced as compared with its fundamental counterpart $\mathcal{J}$. We find that $\widetilde{\mathcal{J}} / \mathcal{J} \gtrsim 1$ will hold if $E$ is below the upper limit $E_{0} \simeq$ $\Delta_{21} \cos 2 \theta_{12} /\left(\sqrt{2} G_{\mathrm{F}} N_{e}\right) \lesssim 0.3 \mathrm{GeV}$ in a realistic oscillation experiment. In particular, we find that $\widetilde{\mathcal{J}} / \mathcal{J}$ peaks at the resonance energy

$$
E_{*} \simeq \frac{\Delta_{21}}{2 \sqrt{2} G_{\mathrm{F}} N_{e}}\left[\cos 2 \theta_{12}\left(1+\sin ^{2} \theta_{13}\right)+\alpha \sin ^{2} 2 \theta_{12}\right]
$$

which is about $0.14 \mathrm{GeV}$ ( or $0.12 \mathrm{GeV}$ ) for $\Delta_{31}>0$ (or $\Delta_{31}<0$ ), corresponding to the normal (or inverted) neutrino mass ordering. Accordingly, we arrive at the maximum value

$$
\frac{\widetilde{\mathcal{J}}_{*}}{\mathcal{J}} \simeq \frac{1}{\sin 2 \theta_{12}}\left[1+\alpha \cos 2 \theta_{12}\left(1+\sin ^{2} \theta_{13}\right)+\text { smaller terms }\right]
$$

which is roughly $110 \%$ (or $107 \%$ ) for $\Delta_{31}>0$ (or $\Delta_{31}<0$ ). As for an antineutrino beam, $\tilde{\mathcal{J}} / \mathcal{J}$ decreases monotonically in the $E \lesssim 1 \mathrm{GeV}$ region and thus does not undergo any resonances. In this sense one may draw the conclusion that a medium-baseline neutrino oscillation experiment with $E$ being in the range $E_{*} \lesssim E \lesssim 2 E_{*}$ should be able to explore leptonic $\mathrm{CP}$ violation with little matter-induced suppression. ${ }^{3}$

In section 3 we concentrate on a geometrical description of leptonic $\mathrm{CP}$ violation in matter and make some analytical approximations for this intuitive and useful language. Namely, we show how the three Dirac unitarity triangles (UTs) in the complex plane [29], ${ }^{4}$ defined through the orthogonality relations

$$
\begin{array}{ll}
\triangle_{e}: & U_{\mu 1} U_{\tau 1}^{*}+U_{\mu 2} U_{\tau 2}^{*}+U_{\mu 3} U_{\tau 3}^{*}=0, \\
\triangle_{\mu}: & U_{\tau 1} U_{e 1}^{*}+U_{\tau 2} U_{e 2}^{*}+U_{\tau 3} U_{e 3}^{*}=0, \\
\triangle_{\tau}: & U_{e 1} U_{\mu 1}^{*}+U_{e 2} U_{\mu 2}^{*}+U_{e 3} U_{\mu 3}^{*}=0,
\end{array}
$$

are modified (either enlarged or suppressed) by terrestrial matter effects in a low-energy medium-baseline neutrino oscillation experiment. We find that the third side of each UT (i.e., $U_{\mu 3} U_{\tau 3}^{*}, U_{\tau 3} U_{e 3}^{*}$ or $U_{e 3} U_{\mu 3}^{*}$ ) is essentially insensitive to the matter-induced corrections when the neutrino beam energy $E$ is low, but the other two sides - both their sizes and orientations - can get appreciable corrections. Besides some new and useful analytical

\footnotetext{
${ }^{3}$ Note that Minakata and Nunokawa have discussed a similar possibility and obtained the leading-order analytical result of $E_{*}$ in ref. [28]. In comparison, our analytical result in eq. (1.3) has a much higher degree of accuracy and thus the new result in eq. (1.4) can explain the sensitivity of $\widetilde{\mathcal{J}}_{*} / \mathcal{J}$ to the neutrino mass ordering.

${ }^{4}$ The other three unitarity triangles (defined as $\triangle_{1}, \triangle_{2}$ and $\triangle_{3}$ ), the so-called Majorana UTs [30, 31], will not be discussed here because they have nothing to do with leptonic $\mathrm{CP}$ and $\mathrm{T}$ violation in normal neutrino-neutrino and antineutrino-antineutrino oscillations.
} 
results to be obtained in a reasonably good approximation, a numerical illustration of the real shapes of the effective Dirac UTs in matter (denoted as $\widetilde{\triangle}_{e}, \widetilde{\triangle}_{\mu}$ and $\widetilde{\triangle}_{\tau}$ ) changing with $E$ will also be presented.

In section 4 we aim to combine our new results about $\widetilde{\mathcal{J}}$ and $\widetilde{\triangle}_{\alpha}($ for $\alpha=e, \mu, \tau)$ with the probabilities of neutrino oscillations in matter. In particular, the effective probabilities $\widetilde{P}\left(\nu_{\mu} \rightarrow \nu_{e}\right)$ and $\widetilde{P}\left(\bar{\nu}_{\mu} \rightarrow \bar{\nu}_{e}\right)$ are expanded in the whole $E \lesssim 1 \mathrm{GeV}$ region with the help of the small quantities $\alpha$ and $\beta$. We show that our analytical approximations are numerically more accurate than those made by Freund in this energy region, and thus they are particularly applicable for the study of leptonic CP violation in the low-energy MOMENT, ESS $\nu$ SM and T2K oscillation experiments. We also affirm that a mediumbaseline neutrino oscillation experiment with the beam energy $E$ lying in the $E_{*} \lesssim E \lesssim 2 E_{*}$ range is capable of exploring leptonic $\mathrm{CP}$ violation with little matter-induced suppression.

\section{The matter-enhanced Jarlskog parameter}

Given the effective neutrino masses $\widetilde{m}_{i}$ and the effective lepton flavor mixing matrix $\widetilde{U}$ which have accommodated the matter-induced corrections to $m_{i}$ and $U$, the effective Hamiltonian responsible for the propagation of a neutrino beam in matter can be written as $[21,22]$

$$
\widetilde{\mathcal{H}}_{\text {eff }}=\frac{1}{2 E}\left[\widetilde{U}\left(\begin{array}{ccc}
\widetilde{m}_{1}^{2} & 0 & 0 \\
0 & \widetilde{m}_{2}^{2} & 0 \\
0 & 0 & \widetilde{m}_{3}^{2}
\end{array}\right) \widetilde{U}^{\dagger}\right]=\frac{1}{2 E}\left[U\left(\begin{array}{ccc}
m_{1}^{2} & 0 & 0 \\
0 & m_{2}^{2} & 0 \\
0 & 0 & m_{3}^{2}
\end{array}\right) U^{\dagger}+\left(\begin{array}{ccc}
A & 0 & 0 \\
0 & 0 & 0 \\
0 & 0 & 0
\end{array}\right)\right],
$$

in which $A=2 \sqrt{2} G_{\mathrm{F}} N_{e} E$ denotes the charged-current contribution to the coherent $\nu_{e} e^{-}$ forward scattering in matter. When a constant terrestrial matter profile is concerned, as in the present work, eq. (2.1) allows one to derive the following relation between the fundamental Jarlskog invariant $\mathcal{J}$ and its matter-corrected counterpart $\widetilde{\mathcal{J}}$ :

$$
\frac{\widetilde{\mathcal{J}}}{\mathcal{J}}=\left|\frac{\widetilde{U}_{e 1}}{U_{e 1}}\right|\left|\frac{\widetilde{U}_{e 2}}{U_{e 2}}\right|\left|\frac{\widetilde{U}_{e 3}}{U_{e 3}}\right|=\frac{\Delta_{21} \Delta_{31} \Delta_{32}}{\widetilde{\Delta}_{21} \widetilde{\Delta}_{31} \widetilde{\Delta}_{32}},
$$

which is a reflection of both the Naumov relation [25-27] and the Toshev relation [32]. The latter means $\sin 2 \widetilde{\theta}_{23} \sin \widetilde{\delta}=\sin 2 \theta_{23} \sin \delta$ in the standard parametrization of $U$ and $\widetilde{U}$. Namely, ${ }^{5}$

$$
U=\left(\begin{array}{ccc}
U_{e 1} & U_{e 2} & U_{e 3} \\
U_{\mu 1} & U_{\mu 2} & U_{\mu 3} \\
U_{\tau 1} & U_{\tau 2} & U_{\tau 3}
\end{array}\right)=\left(\begin{array}{ccc}
c_{12} c_{13} & s_{12} c_{13} & s_{13} e^{-\mathrm{i} \delta} \\
-s_{12} c_{23}-c_{12} s_{13} s_{23} e^{\mathrm{i} \delta} & c_{12} c_{23}-s_{12} s_{13} s_{23} e^{\mathrm{i} \delta} & c_{13} s_{23} \\
s_{12} s_{23}-c_{12} s_{13} c_{23} e^{\mathrm{i} \delta} & -c_{12} s_{23}-s_{12} s_{13} c_{23} e^{\mathrm{i} \delta} & c_{13} c_{23}
\end{array}\right)
$$

with $c_{i j} \equiv \cos \theta_{i j}$ and $s_{i j} \equiv \sin \theta_{i j}$ (for $i j=12,13,23$ ). The parametrization of $\widetilde{U}$ is exactly the same as that of $U$ in eq. (2.3), and hence one may obtain $\mathcal{J}=c_{12} s_{12} c_{13}^{2} s_{13} c_{23} s_{23} \sin \delta$

\footnotetext{
${ }^{5}$ For the sake of simplicity, we have omitted the Majorana CP-violating phases of massive neutrinos in this parametrization simply because they have nothing to do with neutrino oscillations under discussion.
} 
and the same expression of $\widetilde{\mathcal{J}}$ as a function of $\widetilde{\theta}_{12}, \widetilde{\theta}_{13}, \widetilde{\theta}_{23}$ and $\widetilde{\delta}$. Note, however, that eq. (2.2) is actually a parametrization-independent result. We shall use it to establish an approximate but more transparent relationship between $\mathcal{J}$ and $\widetilde{\mathcal{J}}$ later on.

In fact, the exact relations between $\widetilde{m}_{i}^{2}$ and $m_{i}^{2}$ (for $i=1,2,3$ ) have been derived by several authors with the help of eq. (2.1) [33-35], but only the normal neutrino mass ordering (i.e., $\Delta_{31}>0$ ) was assumed in those works. Here we consider both normal and inverted (i.e., $\Delta_{31}<0$ ) neutrino mass hierarchies. To be explicit, we have

$$
\begin{aligned}
& \widetilde{\Delta}_{21}=\frac{2}{3} \sqrt{x^{2}-3 y} \sqrt{3\left(1-z^{2}\right)} \\
& \widetilde{\Delta}_{31}=\frac{1}{3} \sqrt{x^{2}-3 y}\left[3 z+\sqrt{3\left(1-z^{2}\right)}\right], \\
& \widetilde{\Delta}_{32}=\frac{1}{3} \sqrt{x^{2}-3 y}\left[3 z-\sqrt{3\left(1-z^{2}\right)}\right]
\end{aligned}
$$

in the $\Delta_{31}>0$ case; or

$$
\begin{aligned}
& \widetilde{\Delta}_{21}=\frac{1}{3} \sqrt{x^{2}-3 y}\left[3 z-\sqrt{3\left(1-z^{2}\right)}\right], \\
& \widetilde{\Delta}_{31}=-\frac{2}{3} \sqrt{x^{2}-3 y} \sqrt{3\left(1-z^{2}\right)}, \\
& \widetilde{\Delta}_{32}=-\frac{1}{3} \sqrt{x^{2}-3 y}\left[3 z+\sqrt{3\left(1-z^{2}\right)}\right]
\end{aligned}
$$

in the $\Delta_{31}<0$ case, where

$$
\begin{aligned}
& x=\Delta_{31}(1+\alpha+\beta) \\
& y=\Delta_{31}^{2}\left[\alpha+\beta\left(\left|U_{e 1}\right|^{2}+\left|U_{e 2}\right|^{2}\right)+\alpha \beta\left(1-\left|U_{e 2}\right|^{2}\right)\right], \\
& z=\cos \left[\frac{1}{3} \arccos \frac{2 x^{3}-9 x y+27 \Delta_{31}^{3} \alpha \beta\left|U_{e 1}\right|^{2}}{2 \sqrt{\left(x^{2}-3 y\right)^{3}}}\right]
\end{aligned}
$$

with the definitions $\alpha \equiv \Delta_{21} / \Delta_{31}$ and $\beta \equiv A / \Delta_{31}$. When an antineutrino beam is taken into account, the corresponding oscillation behaviors depend on $\widetilde{U}^{*}$ and $-A$. In this case the above formulas remain valid but the replacements $U \rightarrow U^{*}$ and $A \rightarrow-A$ (i.e., $\mathcal{J} \rightarrow-\mathcal{J}$ and $\beta \rightarrow-\beta$ ) are required. Eq. (2.2) tells us that both $\mathcal{J}$ and $\widetilde{\mathcal{J}}$ flip their signs in the above replacements, and thus their ratio remains positive.

Although eqs. (2.4)-(2.6) are exact, they are unable to reveal the dependence of $\widetilde{\Delta}_{i j}$ on $\Delta_{i j}$ in a transparent way. It is therefore important to make reasonable analytical approximations in this connection, so as to simplify the relations between $\widetilde{\Delta}_{i j}$ and $\Delta_{i j}$. The remarkable analytical approximations made by Freund [15] have been popularly applied to the studies of various long- or medium-baseline neutrino oscillation experiments with $E \gtrsim 0.5 \mathrm{GeV} .{ }^{6}$ Given the fact that $7.02 \times 10^{-5} \mathrm{eV}^{2} \leq \Delta_{21} \leq 8.09 \times 10^{-5} \mathrm{eV}^{2}$ holds at the $3 \sigma$

\footnotetext{
${ }^{6}$ See, also, the analytical expansions made in refs. $[16,24,36]$. When the unitarity of the $3 \times 3$ PMNS matrix $U$ is directly or indirectly violated in the presence of light or heavy sterile neutrinos, the similar analytical expansions of neutrino oscillation probabilities have been done by Li and Luo [37].
} 


\begin{tabular}{|ccccc|}
\hline & \multicolumn{2}{c|}{ Normal mass ordering (NMO) } & \multicolumn{2}{l|}{ Inverted mass ordering (IMO) } \\
\hline & best-fit & $3 \sigma$ range & best-fit & $3 \sigma$ range \\
\hline$\theta_{12}$ & $33.48^{\circ}$ & $31.29^{\circ}-35.91^{\circ}$ & $33.48^{\circ}$ & $31.29^{\circ}-35.91^{\circ}$ \\
$\theta_{13}$ & $8.50^{\circ}$ & $7.85^{\circ}-9.10^{\circ}$ & $8.51^{\circ}$ & $7.87^{\circ}-9.11^{\circ}$ \\
$\theta_{23}$ & $42.3^{\circ}$ & $38.2^{\circ}-53.3^{\circ}$ & $49.5^{\circ}$ & $38.6^{\circ}-53.3^{\circ}$ \\
$\delta$ & $306^{\circ}$ & $0^{\circ}-360^{\circ}$ & $254^{\circ}$ & $0^{\circ}-360^{\circ}$ \\
\hline$\frac{\Delta_{21}}{10^{-5} \mathrm{eV}^{2}}$ & 7.50 & $7.02-8.09$ & 7.50 & $7.02-8.09$ \\
$\frac{\Delta_{31}}{10^{-3} \mathrm{eV}^{2}}$ & 2.457 & $2.317-2.607$ & -2.374 & $-2.520--2.226$ \\
\hline
\end{tabular}

Table 1. The best-fit values and $3 \sigma$ ranges of six neutrino oscillation parameters from a global fit of current experimental data [4].

confidence level [4] and the dependence of terrestrial matter effects on the neutrino beam energy $E$ can be effectively expressed as $A \simeq 2.28 \times 10^{-4} \mathrm{eV}^{2}(E / \mathrm{GeV})$ for a realistic ongoing or upcoming neutrino oscillation experiment [38], ${ }^{7}$ the limit $E \gtrsim 0.5 \mathrm{GeV}$ is essentially equivalent to the requirement $|\alpha|<|\beta|$.

But we stress that the case of $|\alpha| \gtrsim|\beta|$ is also interesting in neutrino phenomenology, especially in the aspect of probing leptonic $\mathrm{CP}$ and $\mathrm{T}$ violation in a low-energy mediumbaseline oscillation experiment [28]. In fact, there will be no way to obtain $\widetilde{\mathcal{J}} / \mathcal{J} \gtrsim 1$ if the neutrino beam energy $E$ is higher than about $0.5 \mathrm{GeV}$. To see this point, we calculate the ratio of $\widetilde{\mathcal{J}}$ to $\mathcal{J}$ by using eqs. (2.2)-(2.6) and inputting the best-fit values of $\Delta_{21}, \Delta_{31}, \theta_{12}$ and $\theta_{13}$ listed in table 1 [4]. Allowing $E$ to vary from 0 to $100 \mathrm{GeV}$, we plot the numerical change of $\widetilde{\mathcal{J}} / \mathcal{J}$ with $E$ in figure 1, where both the neutrino (with $A$ ) and antineutrino (with $-A$ ) beams are considered, together with both the normal $\left(\Delta_{31}>0\right)$ and inverted $\left(\Delta_{31}<0\right)$ neutrino mass hierarchies. Some observations and discussions are in order.

(1) Except the extreme case of $\mathcal{J}=0$ (i.e., $\delta=0$ or $\pi$ ) which makes the ratio of $\tilde{\mathcal{J}}$ to $\mathcal{J}$ nonsense, the profile of $\widetilde{\mathcal{J}} / \mathcal{J}$ changing with $E$ is stable and independent of the value of $\theta_{23}$ and the large uncertainties of $\delta$ itself. In all the four possibilities shown in figure 1 , the size of $\widetilde{\mathcal{J}} / \mathcal{J}$ goes down quickly when $E$ becomes larger than about $0.5 \mathrm{GeV}$. As for the case of an antineutrino beam plus the normal mass hierarchy, $\widetilde{\mathcal{J}} / \mathcal{J}$ decreases in a monotonic way and does not develop any maxima or minima. In comparison, $\widetilde{\mathcal{J}} / \mathcal{J}$ can have one maximum in the case of a neutrino beam plus the inverted mass hierarchy, or one maximum and one minimum in the case of an antineutrino beam plus the inverted mass hierarchy, or two maxima and one minimum in the case of a neutrino beam plus the normal mass hierarchy. But we are mainly interested in the peaks of $\widetilde{\mathcal{J}} / \mathcal{J}$ in the latter three cases, where the corresponding

\footnotetext{
${ }^{7}$ To be more specific, the "matter" parameter $A$ is given as $A \simeq 1.52 \times 10^{-4} \mathrm{eV}^{2} Y_{e}\left(\rho / \mathrm{g} / \mathrm{cm}^{3}\right)(E / \mathrm{GeV}) \simeq$ $2.28 \times 10^{-4} \mathrm{eV}^{2}(E / \mathrm{GeV})$, where $Y_{e} \simeq 0.5$ is the electron fraction and $\rho \simeq 3 \mathrm{~g} / \mathrm{cm}^{3}$ is the typical matter density for a neutrino trajectory through the Earth's crust.
} 

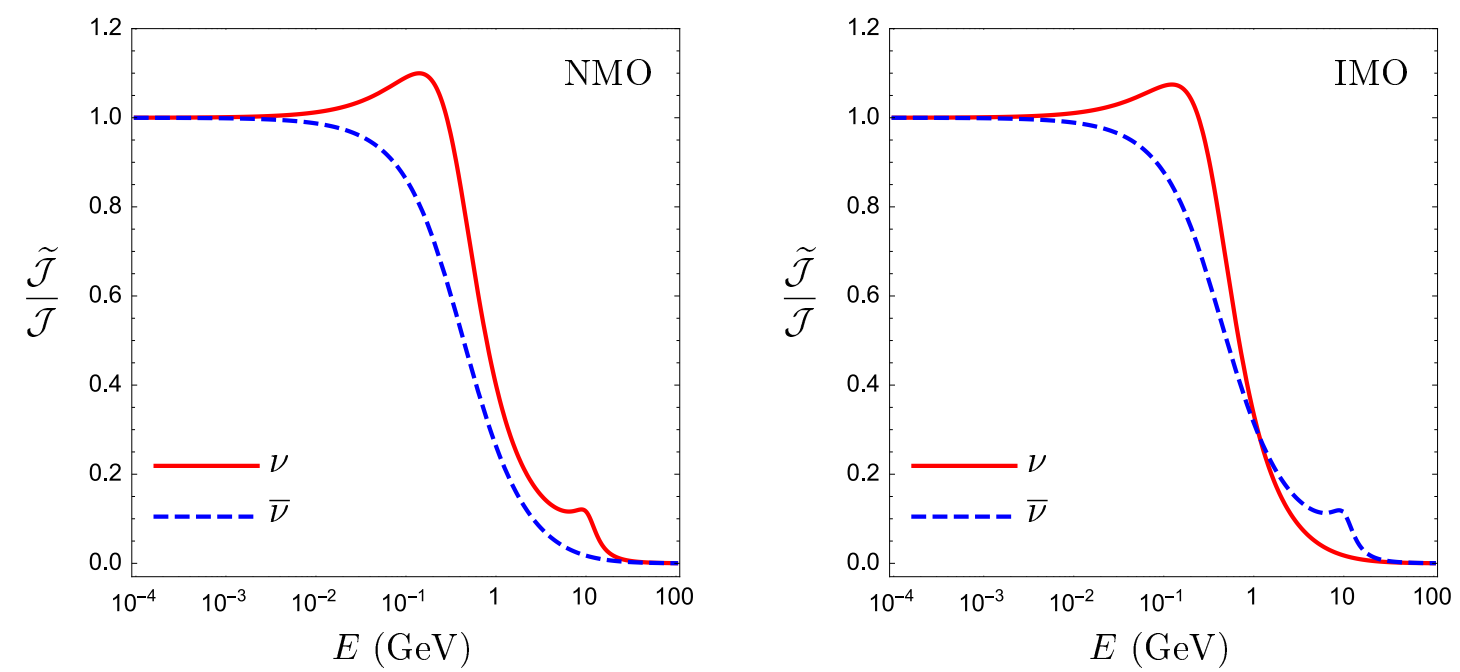

Figure 1. The ratio of the matter-corrected Jarlskog invariant $\widetilde{\mathcal{J}}$ to its fundamental counterpart $\mathcal{J}$ as a function of the neutrino ( $\nu$ with $A$ ) or antineutrino $(\bar{\nu}$ with $-A$ ) beam energy $E$ in the case of a normal mass ordering (NMO, left panel) or an inverted mass ordering (IMO, right panel). Here the best-fit values of $\Delta_{21}, \Delta_{31}, \theta_{12}$ and $\theta_{13}$ [4] have been input.

values of $E$ are summarized as follows: ${ }^{8}$

$$
\begin{aligned}
& \nu \text { beam }\left(\Delta_{31}>0\right): E_{*} \simeq 0.140 \mathrm{GeV}, \frac{\widetilde{\mathcal{J}}_{*}}{\mathcal{J}} \simeq 1.10 ; \quad E_{*}^{\prime} \simeq 8.906 \mathrm{GeV}, \frac{\widetilde{\mathcal{J}}_{*}^{\prime}}{\mathcal{J}} \simeq 0.12 ; \\
& \nu \text { beam }\left(\Delta_{31}<0\right): E_{*} \simeq 0.123 \mathrm{GeV}, \frac{\widetilde{\mathcal{J}}_{*}}{\mathcal{J}} \simeq 1.07 ; \\
& \bar{\nu} \operatorname{beam}\left(\Delta_{31}<0\right): E_{*}^{\prime} \simeq 8.828 \mathrm{GeV}, \frac{\widetilde{\mathcal{J}}_{*}^{\prime}}{\mathcal{J}} \simeq 0.12 .
\end{aligned}
$$

Of course, the suppressed peaks with $\tilde{\mathcal{J}}_{*}^{\prime} / \mathcal{J} \simeq 0.12$ are not within the scope of our interest in this work, because the corresponding beam energies are far above $1 \mathrm{GeV}$.

(2) But a suppressed peak $\tilde{\mathcal{J}}_{*}^{\prime} / \mathcal{J} \simeq 0.12$ and its resonance energy $E_{*}^{\prime}$ can be well understood by following the analytical approximations made in ref. [15] for $E \gtrsim$ $1 \mathrm{GeV}$. Namely,

$$
\frac{\tilde{\mathcal{J}}}{\mathcal{J}} \simeq \frac{\Delta_{21}}{A \cos ^{2} \theta_{13} \sqrt{\beta^{2}-2 \beta \cos 2 \theta_{13}+1}},
$$

in which $\beta= \pm A / \Delta_{31}$ correspond to the neutrino and antineutrino beams, respectively. We find that this ratio peaks at

$$
\beta_{*}^{\prime}=\frac{ \pm A_{*}^{\prime}}{\Delta_{31}} \simeq \frac{3 \cos 2 \theta_{13}+\sqrt{1-9 \sin ^{2} 2 \theta_{13}}}{4}
$$

\footnotetext{
${ }^{8}$ In the case of a neutrino (or antineutrino) beam with the normal (or inverted) mass ordering, the minimum of $\widetilde{\mathcal{J}} / \mathcal{J}$ is about 0.116 (or 0.113 ) appearing at $E \simeq 6.462 \mathrm{GeV}$ (or $6.172 \mathrm{GeV}$ ). The magnitude of such an extreme is actually similar to the suppressed peak $\widetilde{\mathcal{J}}_{*}^{\prime} / \mathcal{J} \simeq 0.12$ at $E_{*}^{\prime} \simeq 8.906 \mathrm{GeV}$ (or $\left.8.828 \mathrm{GeV}\right)$.
} 

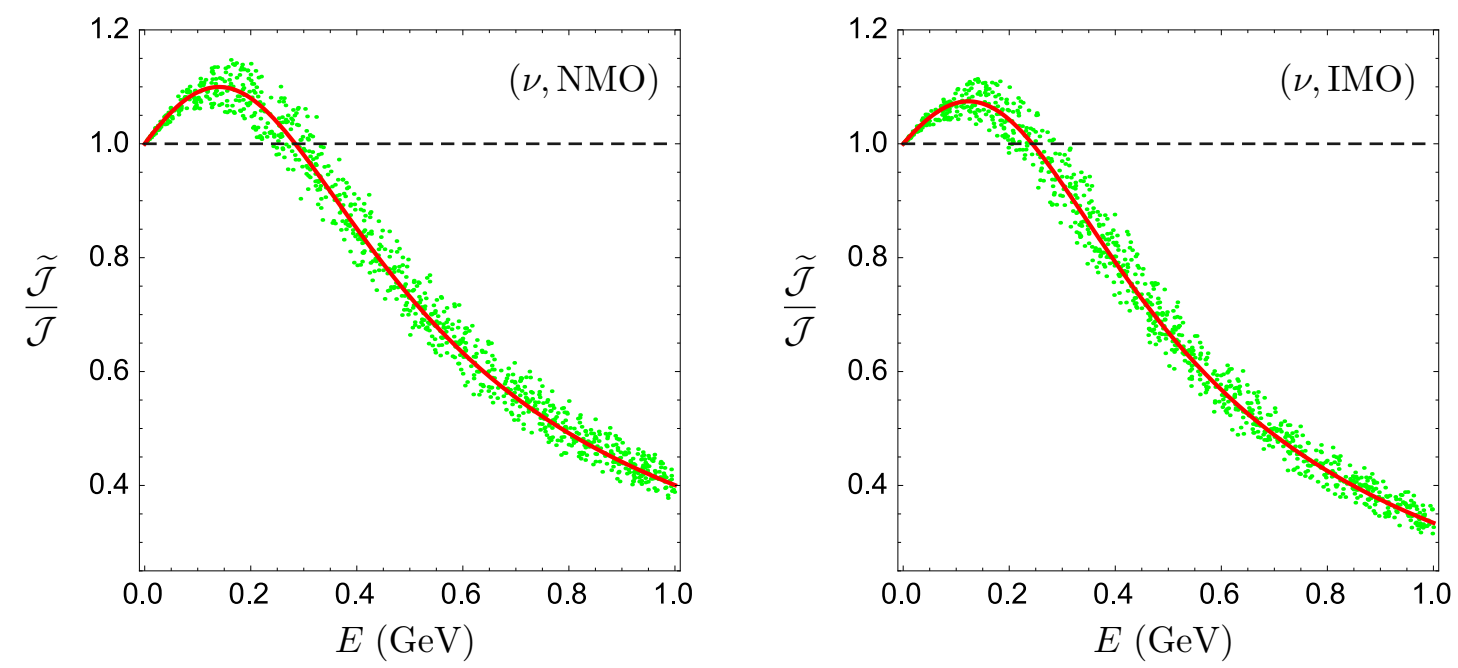

Figure 2. The ratio $\widetilde{\mathcal{J}} / \mathcal{J}$ as a function of the neutrino beam energy $E$ in the normal or inverted mass ordering case. Here the red curve and the green band correspond to the inputs of the best-fit values and $3 \sigma$ ranges of $\Delta_{21}, \Delta_{31}, \theta_{12}$ and $\theta_{13}$ [4], respectively.

with $A_{*}^{\prime}=2 \sqrt{2} G_{\mathrm{F}} N_{e} E_{*}^{\prime}$, where the smallness of $\theta_{13}$ has been taken into account. Now that $\beta_{*}^{\prime}$ itself is positive, the plus (or minus) sign in front of $A_{*}^{\prime}$ in eq. (2.9) must correspond to the neutrino (or antineutrino) beam with the normal (or inverted) mass ordering. Given the best-fit value of $\theta_{13}$ in table 1 , it is straightforward to obtain $E_{*}^{\prime} \simeq 9.02 \mathrm{GeV}$ in the $\Delta_{13}>0$ case or $E_{*}^{\prime} \simeq 8.71 \mathrm{GeV}$ in the $\Delta_{13}<0$ case. Such approximate results are in agreement with the exact numerical results shown in eq. (2.7) to a reasonably good degree of accuracy.

From now on let us concentrate on the first (matter-enhanced) peak $\widetilde{\mathcal{J}}_{*} / \mathcal{J}>1$ around $E_{*} \in(0.1,0.2) \mathrm{GeV}$ in figure 1 and understand why it appears in an approximate but more transparent way. Figure 2 is a clearer illustration of this peak, where the $3 \sigma$ ranges of $\Delta_{21}$, $\Delta_{31}, \theta_{12}$ and $\theta_{13}$ are also taken into account. One can see that the numerical uncertainties associated with the four input parameters do not change the lineshape of $\widetilde{\mathcal{J}} / \mathcal{J}$, implying that our analytical approximations to be made below will keep valid when the relevant neutrino oscillation parameters are measured to a much higher degree of accuracy in the near future. In the low-energy region under consideration the magnitude of $\beta$ is comparable with or smaller than that of $\alpha$, and thus both of them can serve for the small expansion parameters in our analytical approximations for $\widetilde{\Delta}_{21}, \widetilde{\Delta}_{31}$ and $\widetilde{\Delta}_{32}$. We first consider the neutrino beam. A tedious but straightforward calculation leads us to the results

$$
\begin{aligned}
\sqrt{x^{2}-3 y} \simeq & \Delta_{31}\left[1-\frac{1}{2} \alpha-\frac{1}{2}\left(1-3\left|U_{e 3}\right|^{2}\right) \beta+\frac{3}{8} \alpha^{2}-\frac{3}{4}\left(\left|U_{e 1}\right|^{2}-\left|U_{e 2}\right|^{2}\right) \alpha \beta\right. \\
& \left.+\frac{3}{8}\left(1+2\left|U_{e 3}\right|^{2}\right) \beta^{2}\right], \\
z & \simeq 1-\frac{3}{8} \alpha^{2}+\frac{3}{4}\left(\left|U_{e 1}\right|^{2}-\left|U_{e 2}\right|^{2}\right) \alpha \beta-\frac{3}{8}\left(1-2\left|U_{e 3}\right|^{2}\right) \beta^{2}, \\
\sqrt{3\left(1-z^{2}\right)} \simeq & \frac{3}{2} \epsilon\left(1+\frac{1}{2} \alpha+\frac{1}{2} \beta\right)
\end{aligned}
$$


for the $\Delta_{31}>0$ case; and

$$
\begin{aligned}
\sqrt{x^{2}-3 y} \simeq & -\Delta_{31}\left[1-\frac{1}{2} \alpha-\frac{1}{2}\left(1-3\left|U_{e 3}\right|^{2}\right) \beta+\frac{3}{8} \alpha^{2}-\frac{3}{4}\left(\left|U_{e 1}\right|^{2}-\left|U_{e 2}\right|^{2}\right) \alpha \beta\right. \\
& \left.+\frac{3}{8}\left(1+2\left|U_{e 3}\right|^{2}\right) \beta^{2}\right], \\
z \simeq & \frac{1}{2}+\frac{3}{4} \epsilon\left(1+\frac{1}{2} \alpha+\frac{1}{2} \beta\right)-\frac{3}{16} \alpha^{2}+\frac{3}{8}\left(\left|U_{e 1}\right|^{2}-\left|U_{e 2}\right|^{2}\right) \alpha \beta \\
& -\frac{3}{16}\left(1-2\left|U_{e 3}\right|^{2}\right) \beta^{2} \\
\sqrt{3\left(1-z^{2}\right)} \simeq & \frac{3}{2}-\frac{3}{4} \epsilon\left(1+\frac{1}{2} \alpha+\frac{1}{2} \beta\right)-\frac{9}{16} \alpha^{2}+\frac{9}{8}\left(\left|U_{e 1}\right|^{2}-\left|U_{e 2}\right|^{2}\right) \alpha \beta \\
& -\frac{9}{16}\left(1-2\left|U_{e 3}\right|^{2}\right) \beta^{2}
\end{aligned}
$$

for the $\Delta_{31}<0$ case, where

$$
\epsilon \equiv \sqrt{\alpha^{2}-2\left(\left|U_{e 1}\right|^{2}-\left|U_{e 2}\right|^{2}\right) \alpha \beta+\left(1-\left|U_{e 3}\right|^{2}\right)^{2} \beta^{2}}
$$

is a small parameter, and the smallness of $\left|U_{e 3}\right|$ is already implied. Then we obtain the effective neutrino mass-squared differences from eq. (2.4) or eq. (2.5):

$$
\begin{aligned}
& \widetilde{\Delta}_{21} \simeq \Delta_{31}\left(1+\frac{3}{2}\left|U_{e 3}\right|^{2} \beta\right) \epsilon, \\
& \widetilde{\Delta}_{31} \simeq \Delta_{31}\left[1-\frac{1}{2} \alpha-\frac{1}{2}\left(1-3\left|U_{e 3}\right|^{2}\right) \beta+\frac{1}{2} \epsilon+\frac{3}{4}\left|U_{e 3}\right|^{2} \beta \epsilon+\frac{3}{2}\left|U_{e 3}\right|^{2} \beta^{2}\right], \\
& \widetilde{\Delta}_{32} \simeq \Delta_{31}\left[1-\frac{1}{2} \alpha-\frac{1}{2}\left(1-3\left|U_{e 3}\right|^{2}\right) \beta-\frac{1}{2} \epsilon-\frac{3}{4}\left|U_{e 3}\right|^{2} \beta \epsilon+\frac{3}{2}\left|U_{e 3}\right|^{2} \beta^{2}\right],
\end{aligned}
$$

for the $\Delta_{31}>0$ case; or

$$
\begin{aligned}
& \widetilde{\Delta}_{21} \simeq-\Delta_{31}\left(1+\frac{3}{2}\left|U_{e 3}\right|^{2} \beta\right) \epsilon, \\
& \widetilde{\Delta}_{31} \simeq \Delta_{31}\left[1-\frac{1}{2} \alpha-\frac{1}{2}\left(1-3\left|U_{e 3}\right|^{2}\right) \beta-\frac{1}{2} \epsilon-\frac{3}{4}\left|U_{e 3}\right|^{2} \beta \epsilon+\frac{3}{2}\left|U_{e 3}\right|^{2} \beta^{2}\right], \\
& \widetilde{\Delta}_{32} \simeq \Delta_{31}\left[1-\frac{1}{2} \alpha-\frac{1}{2}\left(1-3\left|U_{e 3}\right|^{2}\right) \beta+\frac{1}{2} \epsilon+\frac{3}{4}\left|U_{e 3}\right|^{2} \beta \epsilon+\frac{3}{2}\left|U_{e 3}\right|^{2} \beta^{2}\right],
\end{aligned}
$$

for the $\Delta_{31}<0$ case. Given the standard parametrization of the PMNS mixing matrix $U$ in eq. (2.3), the small parameter $\epsilon$ in eq. (2.12) can be reexpressed as

$$
\epsilon=\sqrt{\alpha^{2}-2 \alpha \beta \cos 2 \theta_{12} \cos ^{2} \theta_{13}+\beta^{2} \cos ^{4} \theta_{13}},
$$

so its magnitude is apparently of $\mathcal{O}(\alpha)$ or $\mathcal{O}(\beta)$. With the help of eqs. (2.2), (2.13) and (2.14), we arrive at the approximate analytical results for the ratio of $\widetilde{\mathcal{J}}$ to $\mathcal{J}$ as follows:

$$
\frac{\widetilde{\mathcal{J}}}{\mathcal{J}} \simeq+\frac{\alpha}{\epsilon}(1+\beta) \simeq \frac{\alpha}{\sqrt{\alpha^{2}-2 \alpha \beta \cos 2 \theta_{12}+\beta^{2}}}(1+\beta),
$$


for the $\Delta_{31}>0$ case; or

$$
\frac{\widetilde{\mathcal{J}}}{\mathcal{J}} \simeq-\frac{\alpha}{\epsilon}(1+\beta) \simeq \frac{-\alpha}{\sqrt{\alpha^{2}-2 \alpha \beta \cos 2 \theta_{12}+\beta^{2}}}(1+\beta),
$$

for the $\Delta_{31}<0$ case, in which the terms proportional to $\left|U_{e 3}\right|^{2}=\sin ^{2} \theta_{13}$ in $\epsilon$ have been omitted thanks to the smallness of $\theta_{13}$. Since $\epsilon$ has a minimum value $\epsilon_{*} \simeq|\alpha| \sin 2 \theta_{12}$ at $\beta_{*} \simeq \alpha \cos 2 \theta_{12}$, we expect that the ratio $\tilde{\mathcal{J}} / \mathcal{J}$ takes its maximum value $1 / \sin 2 \theta_{12}$ in the leading-order approximation, no matter whether the neutrino mass ordering is normal or inverted. As for an antineutrino beam, the matter parameter is actually $-A$, and thus the replacement $\beta \rightarrow-\beta$ must be made for the analytical results obtained above. In other words, $\epsilon$ does not develop a minimum value in the antineutrino case - that is why $\widetilde{\mathcal{J}} / \mathcal{J}$ does not undergo any resonances in this case, a conclusion independent of the neutrino mass ordering. So we only concentrate on the neutrino beam in the subsequent discussions.

Let us go beyond the leading-order approximation to calculate the extreme value of $\widetilde{\mathcal{J}} / \mathcal{J}$, which is a function of $\beta$ (or equivalently, the matter parameter $A$ or the neutrino beam energy $E$ ). To do so, we take the first derivative of $\widetilde{\mathcal{J}} / \mathcal{J}$ with respect to the variable $\beta$ in eq. (2.16) or (2.17) and set it to equal zero, and find that such a treatment leads to the same equation in these two cases:

$$
\left[\left(1-\left|U_{e 3}\right|^{2}\right)^{2}+\alpha\left(\left|U_{e 1}\right|^{2}-\left|U_{e 2}\right|^{2}\right)\right] \beta-\left(\left|U_{e 1}\right|^{2}-\left|U_{e 2}\right|^{2}\right) \alpha-\alpha^{2} \simeq 0 .
$$

The solution to eq. (2.18) turns out to be

$$
\beta_{*}=\frac{A_{*}}{\Delta_{31}} \simeq \alpha\left[\cos 2 \theta_{12}\left(1+\sin ^{2} \theta_{13}\right)+\alpha \sin ^{2} 2 \theta_{12}\right]
$$

with $A_{*}=2 \sqrt{2} G_{\mathrm{F}} N_{e} E_{*}$, from which one can easily obtain the resonance energy $E_{*}$ that has been given in eq. (1.3). Substituting eq. (2.19) into eq. (2.16) or (2.17), we immediately arrive at the maximum value of $\widetilde{\mathcal{J}} / \mathcal{J}$ on the resonance:

$$
\frac{\widetilde{\mathcal{J}}_{*}}{\mathcal{J}} \simeq \frac{1}{\sin 2 \theta_{12}}\left[1+\alpha \cos 2 \theta_{12}\left(1+\sin ^{2} \theta_{13}\right)+\frac{1}{2} \alpha^{2} \sin ^{2} 2 \theta_{12}\right]
$$

an interesting and instructive result whose leading and next-to-leading-order parts have been shown in eq. (1.4). Taking the best-fit values of $\theta_{12}, \theta_{13}, \Delta_{21}$ and $\Delta_{31}$ for example, we obtain $E_{*} \simeq 0.140 \mathrm{GeV}$ (or $0.123 \mathrm{GeV}$ ) and $\widetilde{\mathcal{J}}_{*} / \mathcal{J} \simeq 1.10$ (or 1.07) for the normal (or inverted) neutrino mass ordering from the analytical formulas in eqs. (2.19) and (2.20), in good agreement with the more exact numerical results that have been listed in eq. (2.7).

In figure 3 we compare the result of $\widetilde{\mathcal{J}} / \mathcal{J}$ obtained from our analytical approximation made in eq. (2.16) or (2.17) with its exact numerical result by allowing the neutrino beam energy $E$ to vary from zero to $1 \mathrm{GeV}$. We see that the two sets of results agree with each other in a perfect way. In contrast, the numerical result of $\widetilde{\mathcal{J}} / \mathcal{J}$ obtained from Freund's analytical approximation in eq. (2.8) is not so good in the $0.4 \mathrm{GeV} \lesssim E \lesssim 1 \mathrm{GeV}$ range, and it becomes out of control for $E \lesssim 0.4 \mathrm{GeV}$. Hence our analytical approximations stand 

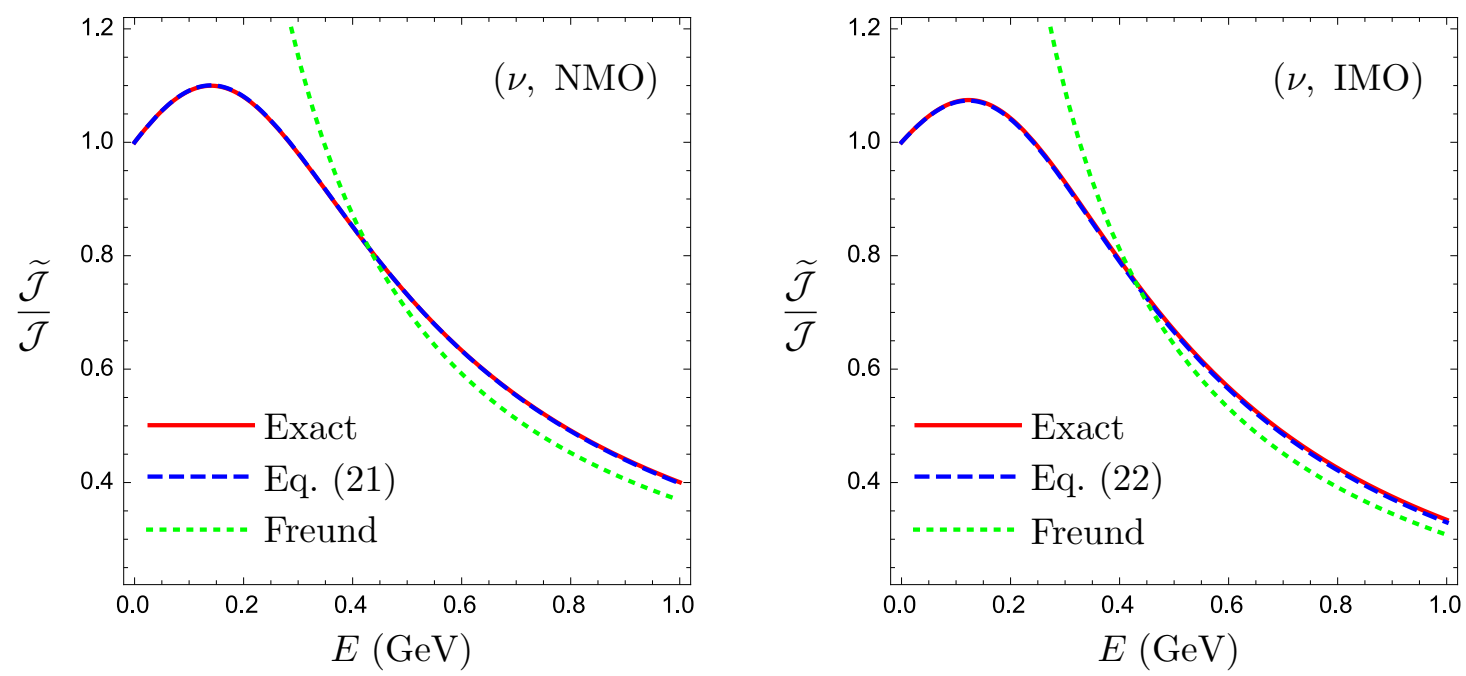

Figure 3. A numerical comparison between the results of $\widetilde{\mathcal{J}} / \mathcal{J}$ obtained from the exact formula in eq. (2.2) (red line) and our analytical approximations in eqs. (2.16) and (2.17) (blue dashed curve) or Freund's approximation in eq. (2.8) (green dotted curve), where $E$ varies from zero to $1 \mathrm{GeV}$, and the best-fit values of $\Delta_{21}, \Delta_{31}, \theta_{12}$ and $\theta_{13}$ [4] have been input.

out as a much better tool of understanding the salient features of the matter-corrected Jarlskog parameter in the $E \lesssim 1 \mathrm{GeV}$ region. In fact, the typical neutrino beam energy of the realistic T2K long-baseline oscillation experiment [5] is about $0.6 \mathrm{GeV}$, just within this region. So one may use the analytical formulas given in the present work to do a reliable phenomenological analysis of $\mathrm{CP}$ violation and the associated matter effects in the T2K experiment.

Given the resonance energy $E_{*}$ in eq. (1.3) and the maximum value $\widetilde{\mathcal{J}}_{*} / \mathcal{J}$ in eq. (1.4), the profiles of $\widetilde{\mathcal{J}} / \mathcal{J}$ in the left and right panels of figure 2 can easily be understood. Simply because the next-to-leading-order terms of $E_{*}$ and $\widetilde{\mathcal{J}}_{*} / \mathcal{J}$ are both proportional to the expansion parameter $\alpha=\Delta_{21} / \Delta_{31} \simeq \pm 1 / 30$, they exhibit a small but appreciable difference in figure 2 with respect to the normal and inverted neutrino mass hierarchies. This observation indicates that even a low-energy neutrino oscillation experiment could have the potential to probe not only the CP- and T-violating effects but also the neutrino mass ordering.

At this point it is worth stressing that the matter-induced amplification or enhancement of $\widetilde{\mathcal{J}}$ under discussion is actually associated with the sensitivity of $\theta_{12}$ to the matterinduced correction. It is well known that $\theta_{13}, \theta_{23}$ and $\delta$ are almost insensitive to terrestrial matter effects (i.e., $\widetilde{\theta}_{13} \simeq \theta_{13}, \widetilde{\theta}_{23} \simeq \theta_{23}$ and $\widetilde{\delta} \simeq \delta$ ) in the $E \lesssim 1 \mathrm{GeV}$ region [24, 28, 39], and hence the first equality in eq. (2.2) leads us to the relation

$$
\frac{\sin 2 \widetilde{\theta}_{12}}{\sin 2 \theta_{12}} \simeq \frac{\widetilde{\mathcal{J}}}{\mathcal{J}} \simeq \frac{|\alpha|}{\epsilon}(1+\beta)
$$

where eq. (2.16) or (2.17) has been taken into account. So the behavior of the ratio of $\sin 2 \widetilde{\theta}_{12}$ to $\sin 2 \theta_{12}$ changing with $E$ is expected to be the same as that of $\widetilde{\mathcal{J}} / \mathcal{J}$ shown in figure 2 . 
Last but not least, let us figure out the upper limit of $E$ which allows $\widetilde{\mathcal{J}} / \mathcal{J} \gtrsim 1$ to hold. For this purpose, we take $\widetilde{\mathcal{J}} / \mathcal{J} \simeq 1$ in eq. (2.16) or (2.17) and then solve this equation. Besides the trivial solution $E=0$, there is a nontrivial solution

$$
E_{0} \simeq \frac{\Delta_{21}}{\sqrt{2} G_{\mathrm{F}} N_{e}}\left[\cos 2 \theta_{12}\left(1+\sin ^{2} \theta_{13}\right)+\alpha\right],
$$

which is valid for both normal and inverted neutrino mass hierarchies. Namely, $\widetilde{\mathcal{J}} / \mathcal{J} \gtrsim 1$ holds for $E \in\left[0, E_{0}\right]$ - the region of $E$ which might be especially interesting for the study of leptonic CP violation in a low-energy medium-baseline neutrino oscillation experiment. If only the leading term in eq. (2.22) is taken into account (i.e., omitting the $\alpha$ term and taking $\sin ^{2} \theta_{13} \simeq 0$ ), we are then left with $E_{0} \simeq \Delta_{21} \cos 2 \theta_{12} /\left(\sqrt{2} G_{\mathrm{F}} N_{e}\right) \lesssim 0.3 \mathrm{GeV}$ by considering $A \simeq 2.28 \times 10^{-4} \mathrm{eV}^{2}(E / \mathrm{GeV})$ and inputting the best-fit values of $\Delta_{21}$ and $\theta_{12}$ [4]. Given the best-fit values and $3 \sigma$ ranges of $\Delta_{21}, \Delta_{31}, \theta_{12}$ and $\theta_{13}$ listed in table 1 , the more accurate results of $E_{0}$ can be obtained from solving $\widetilde{\mathcal{J}} / \mathcal{J}=1$ in eq. (2.2) in a

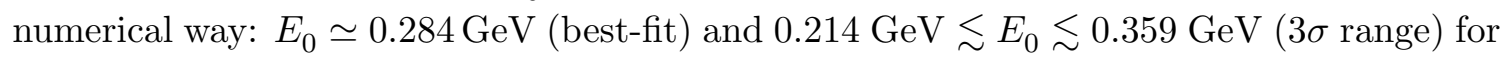
the normal neutrino mass ordering, or $E_{0} \simeq 0.244 \mathrm{GeV}$ (best-fit) and $0.179 \mathrm{GeV} \lesssim E_{0} \lesssim$ $0.312 \mathrm{GeV}$ ( $3 \sigma$ range) for the inverted neutrino mass ordering. These results are consistent with those shown in figure 2 .

Since $E_{0} \simeq 2 E_{*}$ holds as a good approximation, one could consider to set the neutrino beam energy $E$ in the $E_{*} \lesssim E \lesssim 2 E_{*}$ range when designing a realistic medium-baseline oscillation experiment to probe the $\widetilde{\mathcal{J}} / \mathcal{J} \gtrsim 1$ region of $\mathrm{CP}$ violation. In fact, the typical beam energies of the proposed MOMENT [13] and ESS $\nu \mathrm{SM}$ [14] experiments just lie in such an interesting region.

\section{The matter-deformed unitarity triangles}

The three Dirac UTs defined in vacuum in eq. (1.5) have their counterparts in matter, namely,

$$
\begin{array}{ll}
\widetilde{\triangle}_{e}: & \widetilde{U}_{\mu 1} \widetilde{U}_{\tau 1}^{*}+\widetilde{U}_{\mu 2} \widetilde{U}_{\tau 2}^{*}+\widetilde{U}_{\mu 3} \widetilde{U}_{\tau 3}^{*}=0, \\
\widetilde{\triangle}_{\mu}: & \widetilde{U}_{\tau 1} \widetilde{U}_{e 1}^{*}+\widetilde{U}_{\tau 2} \widetilde{U}_{e 2}^{*}+\widetilde{U}_{\tau 3} \widetilde{U}_{e 3}^{*}=0, \\
\widetilde{\triangle}_{\tau}: & \widetilde{U}_{e 1} \widetilde{U}_{\mu 1}^{*}+\widetilde{U}_{e 2} \widetilde{U}_{\mu 2}^{*}+\widetilde{U}_{e 3} \widetilde{U}_{\mu 3}^{*}=0 .
\end{array}
$$

Thanks to the unitarity of $U$ and $\widetilde{U}$, the areas of $\triangle_{\alpha}$ and $\widetilde{\triangle}_{\alpha}$ (for $\alpha=e, \mu, \tau$ ) are equal to $|\mathcal{J}| / 2$ and $|\widetilde{\mathcal{J}}| / 2$, respectively. Hence a change of the ratio $\widetilde{\mathcal{J}} / \mathcal{J}$ with the neutrino beam energy $E$ implies that the three UTs must be deformed by terrestrial matter effects. The exact analytical expressions of the three sides of $\widetilde{\triangle}_{\alpha}$ for a constant matter profile have been derived in ref. [40]. Here we find a more convenient way to reexpress the previous results, ${ }^{9}$ and take into account both normal and inverted neutrino mass hierarchies. ${ }^{10}$ To

\footnotetext{
${ }^{9}$ In the low-energy region under consideration we find that the $U_{\alpha 3} U_{\beta 3}^{*}$ side of $\triangle_{\gamma}$, where the subscripts $\alpha, \beta$ and $\gamma$ run over $e, \mu$ and $\tau$ cyclically, is least sensitive to terrestrial matter effects. Hence it is appropriate to express the other two sides in matter as $\widetilde{U}_{\alpha i} \widetilde{U}_{\beta i}^{*}=c_{1} U_{\alpha i} U_{\beta i}^{*}+c_{2} U_{\alpha 3} U_{\beta 3}^{*}$ (for $i=1$ or 2 ), in which the coefficients $c_{1}$ and $c_{2}$ deviate respectively from 1 and 0 due to the matter-induced corrections.

${ }^{10}$ In this connection only the possibility of a normal neutrino mass hierarchy is discussed analytically and numerically in the literature. The present work improves the previous ones by taking account of both normal and inverted mass hierarchies and shows the phenomenological differences between these two cases.
} 
be specific, we obtain the formulas for a neutrino beam as follows:

$$
\widetilde{\Delta}_{e}:\left\{\begin{array}{l}
\widetilde{U}_{\mu 1} \widetilde{U}_{\tau 1}^{*}=\frac{\left(\Delta_{31}^{\prime}+A\right) \Delta_{21}}{\widetilde{\Delta}_{21} \widetilde{\Delta}_{31}} U_{\mu 1} U_{\tau 1}^{*}-\frac{\left(\Delta_{11}^{\prime}+A\right) \Delta_{32}}{\widetilde{\Delta}_{21} \widetilde{\Delta}_{31}} U_{\mu 3} U_{\tau 3}^{*}, \\
\widetilde{U}_{\mu 2} \widetilde{U}_{\tau 2}^{*}=\frac{\left(\Delta_{32}^{\prime}+A\right) \Delta_{21}}{\widetilde{\Delta}_{21} \widetilde{\Delta}_{32}} U_{\mu 2} U_{\tau 2}^{*}+\frac{\left(\Delta_{22}^{\prime}+A\right) \Delta_{31}}{\widetilde{\Delta}_{21} \widetilde{\Delta}_{32}} U_{\mu 3} U_{\tau 3}^{*}, \\
\widetilde{U}_{\mu 3} \widetilde{U}_{\tau 3}^{*}=\frac{\left(\Delta_{13}^{\prime}+A\right) \Delta_{23}}{\widetilde{\Delta}_{31} \widetilde{\Delta}_{32}} U_{\mu 3} U_{\tau 3}^{*}+\frac{\left(\Delta_{33}^{\prime}+A\right) \Delta_{21}}{\widetilde{\Delta}_{31} \widetilde{\Delta}_{32}} U_{\mu 1} U_{\tau 1}^{*} ;
\end{array}\right.
$$

and

$$
\widetilde{\Delta}_{\mu}:\left\{\begin{array}{l}
\widetilde{U}_{\tau 1} \widetilde{U}_{e 1}^{*}=\frac{\Delta_{31}^{\prime} \Delta_{21}}{\widetilde{\Delta}_{21} \widetilde{\Delta}_{31}} U_{\tau 1} U_{e 1}^{*}-\frac{\Delta_{11}^{\prime} \Delta_{32}}{\widetilde{\Delta}_{21} \widetilde{\Delta}_{31}} U_{\tau 3} U_{e 3}^{*}, \\
\widetilde{U}_{\tau 2} \widetilde{U}_{e 2}^{*}=\frac{\Delta_{32}^{\prime} \Delta_{21}}{\widetilde{\Delta}_{21} \widetilde{\Delta}_{32}} U_{\tau 2} U_{e 2}^{*}+\frac{\Delta_{22}^{\prime} \Delta_{31}}{\widetilde{\Delta}_{21} \widetilde{\Delta}_{32}} U_{\tau 3} U_{e 3}^{*}, \\
\widetilde{U}_{\tau 3} \widetilde{U}_{e 3}^{*}=\frac{\Delta_{13}^{\prime} \Delta_{23}}{\widetilde{\Delta}_{31} \widetilde{\Delta}_{32}} U_{\tau 3} U_{e 3}^{*}+\frac{\Delta_{33}^{\prime} \Delta_{21}}{\widetilde{\Delta}_{31} \widetilde{\Delta}_{32}} U_{\tau 1} U_{e 1}^{*} ;
\end{array}\right.
$$

and

$$
\widetilde{\Delta}_{\tau}:\left\{\begin{array}{l}
\widetilde{U}_{e 1} \widetilde{U}_{\mu 1}^{*}=\frac{\Delta_{31}^{\prime} \Delta_{21}}{\widetilde{\Delta}_{21} \widetilde{\Delta}_{31}} U_{e 1} U_{\mu 1}^{*}-\frac{\Delta_{11}^{\prime} \Delta_{32}}{\widetilde{\Delta}_{21} \widetilde{\Delta}_{31}} U_{e 3} U_{\mu 3}^{*}, \\
\widetilde{U}_{e 2} \widetilde{U}_{\mu 2}^{*}=\frac{\Delta_{32}^{\prime} \Delta_{21}}{\widetilde{\Delta}_{21} \widetilde{\Delta}_{32}} U_{e 2} U_{\mu 2}^{*}+\frac{\Delta_{22}^{\prime} \Delta_{31}}{\widetilde{\Delta}_{21} \widetilde{\Delta}_{32}} U_{e 3} U_{\mu 3}^{*}, \\
\widetilde{U}_{e 3} \widetilde{U}_{\mu 3}^{*}=\frac{\Delta_{13}^{\prime} \Delta_{23}}{\widetilde{\Delta}_{31} \widetilde{\Delta}_{32}} U_{e 3} U_{\mu 3}^{*}+\frac{\Delta_{33}^{\prime} \Delta_{21}}{\widetilde{\Delta}_{31} \widetilde{\Delta}_{32}} U_{e 1} U_{\mu 1}^{*},
\end{array}\right.
$$

where $\Delta_{i j} \equiv m_{i}^{2}-m_{j}^{2}, \widetilde{\Delta}_{i j} \equiv \widetilde{m}_{i}^{2}-\widetilde{m}_{j}^{2}$ and $\Delta_{i j}^{\prime} \equiv m_{i}^{2}-\widetilde{m}_{j}^{2}=\Delta_{i j}+\Delta_{j j}^{\prime}($ for $i, j=1,2,3)$ with $\Delta_{j j}^{\prime}$ being expressed as

$$
\begin{aligned}
& \Delta_{11}^{\prime}=-\frac{1}{3} x+\frac{1}{3} \sqrt{x^{2}-3 y}\left[z+\sqrt{3\left(1-z^{2}\right)}\right], \\
& \Delta_{22}^{\prime}=-\frac{1}{3} x+\frac{1}{3} \sqrt{x^{2}-3 y}\left[z-\sqrt{3\left(1-z^{2}\right)}\right]+\Delta_{21}, \\
& \Delta_{33}^{\prime}=-\frac{1}{3} x-\frac{2}{3} z \sqrt{x^{2}-3 y}+\Delta_{31}
\end{aligned}
$$

in the $\Delta_{31}>0$ case; or

$$
\begin{aligned}
& \Delta_{11}^{\prime}=-\frac{1}{3} x+\frac{1}{3} \sqrt{x^{2}-3 y}\left[z-\sqrt{3\left(1-z^{2}\right)}\right], \\
& \Delta_{22}^{\prime}=-\frac{1}{3} x-\frac{2}{3} z \sqrt{x^{2}-3 y}+\Delta_{21}, \\
& \Delta_{33}^{\prime}=-\frac{1}{3} x+\frac{1}{3} \sqrt{x^{2}-3 y}\left[z+\sqrt{3\left(1-z^{2}\right)}\right]+\Delta_{31}
\end{aligned}
$$

in the $\Delta_{31}<0$ case. Eqs. (3.2)-(3.6) are exact and elegant in showing the corrections of terrestrial matter to the sides of three UTs, but they are unable to give one a ball-park 
feeling of the order of magnitude of such corrections due to the complication of $\widetilde{\Delta}_{i j}$ and $\Delta_{i j}^{\prime}$. Hence it is necessary to make some analytical approximations in order to show the deviation of $\widetilde{\triangle}_{\alpha}$ from $\triangle_{\alpha}$ (for $\left.\alpha=e, \mu, \tau\right)$ in a direct and transparent way.

Note that such an exercise is not only conceptually interesting and intuitive but also helpful for expanding the matter-corrected probabilities of $\nu_{\mu} \rightarrow \nu_{e}$ and $\bar{\nu}_{\mu} \rightarrow \bar{\nu}_{e}$ oscillations in terms of the small parameters $\alpha$ and $\beta$ in the $E \lesssim 1 \mathrm{GeV}$ region, because the CPconserving parts of $\widetilde{P}\left(\nu_{\mu} \rightarrow \nu_{e}\right)$ and $\widetilde{P}\left(\bar{\nu}_{\mu} \rightarrow \bar{\nu}_{e}\right)$ are directly related to the sides of the above effective Dirac UTs. This point will become clear in section 4.

With the help of eqs. (2.10), (2.13) and (3.5), some straightforward calculations lead us to the following approximate expressions in the case of a normal neutrino mass hierarchy:

$$
\begin{aligned}
& \Delta_{11}^{\prime} \simeq-\Delta_{31}\left[\frac{1}{2} \alpha+\frac{1}{2}\left(1-\left|U_{e 3}\right|^{2}\right) \beta-\frac{1}{2} \epsilon-\frac{3}{4}\left|U_{e 3}\right|^{2} \beta \epsilon-\frac{1}{2}\left|U_{e 3}\right|^{2} \beta^{2}\right], \\
& \Delta_{21}^{\prime} \simeq \Delta_{31}\left[\frac{1}{2} \alpha-\frac{1}{2}\left(1-\left|U_{e 3}\right|^{2}\right) \beta+\frac{1}{2} \epsilon+\frac{3}{4}\left|U_{e 3}\right|^{2} \beta \epsilon+\frac{1}{2}\left|U_{e 3}\right|^{2} \beta^{2}\right], \\
& \Delta_{22}^{\prime} \simeq \Delta_{31}\left[\frac{1}{2} \alpha-\frac{1}{2}\left(1-\left|U_{e 3}\right|^{2}\right) \beta-\frac{1}{2} \epsilon-\frac{3}{4}\left|U_{e 3}\right|^{2} \beta \epsilon+\frac{1}{2}\left|U_{e 3}\right|^{2} \beta^{2}\right], \\
& \Delta_{31}^{\prime} \simeq \Delta_{31}\left[1-\frac{1}{2} \alpha-\frac{1}{2}\left(1-\left|U_{e 3}\right|^{2}\right) \beta+\frac{1}{2} \epsilon+\frac{3}{4}\left|U_{e 3}\right|^{2} \beta \epsilon+\frac{1}{2}\left|U_{e 3}\right|^{2} \beta^{2}\right], \\
& \Delta_{32}^{\prime} \simeq \Delta_{31}\left[1-\frac{1}{2} \alpha-\frac{1}{2}\left(1-\left|U_{e 3}\right|^{2}\right) \beta-\frac{1}{2} \epsilon-\frac{3}{4}\left|U_{e 3}\right|^{2} \beta \epsilon+\frac{1}{2}\left|U_{e 3}\right|^{2} \beta^{2}\right],
\end{aligned}
$$

together with $\Delta_{13}^{\prime} \simeq-\Delta_{31}\left(1+\left|U_{e 3}\right|^{2} \beta\right)$ and $\Delta_{33}^{\prime} \simeq-\Delta_{31}\left|U_{e 3}\right|^{2} \beta$. When an inverted neutrino mass ordering is concerned, the corresponding expressions of $\Delta_{i j}^{\prime}$ can simply be obtained from eq. (3.7) with the replacement $\epsilon \rightarrow-\epsilon$. Given eqs. (2.13), (2.14) and (3.7), it is easy to make analytical approximations to the sides of three Dirac UTs in eqs. (3.2)-(3.4). In the case of a normal mass hierarchy, we arrive at

$$
\widetilde{\triangle}_{e}:\left\{\begin{array}{l}
\widetilde{U}_{\mu 1} \widetilde{U}_{\tau 1}^{*} \simeq \frac{\alpha}{\epsilon} U_{\mu 1} U_{\tau 1}^{*}-\frac{1}{2}\left(1-\frac{\alpha-\beta}{\epsilon}\right) U_{\mu 3} U_{\tau 3}^{*}, \\
\widetilde{U}_{\mu 2} \widetilde{U}_{\tau 2}^{*} \simeq \frac{\alpha}{\epsilon} U_{\mu 2} U_{\tau 2}^{*}-\frac{1}{2}\left(1-\frac{\alpha+\beta}{\epsilon}\right) U_{\mu 3} U_{\tau 3}^{*}, \\
\widetilde{U}_{\mu 3} \widetilde{U}_{\tau 3}^{*} \simeq\left(1-2 \beta \sin ^{2} \theta_{13}+\alpha \beta \sin ^{2} \theta_{12}\right) U_{\mu 3} U_{\tau 3}^{*}+\alpha \beta U_{\mu 1} U_{\tau 1}^{*}
\end{array}\right.
$$

and

$$
\widetilde{\triangle}_{\mu}:\left\{\begin{array}{l}
\widetilde{U}_{\tau 1} \widetilde{U}_{e 1}^{*} \simeq \frac{\alpha}{\epsilon} U_{\tau 1} U_{e 1}^{*}-\frac{1}{2}\left(1+\beta-\frac{\alpha+\beta}{\epsilon}\right) U_{\tau 3} U_{e 3}^{*} \\
\widetilde{U}_{\tau 2} \widetilde{U}_{e 2}^{*} \simeq \frac{\alpha}{\epsilon} U_{\tau 2} U_{e 2}^{*}-\frac{1}{2}\left(1+\beta-\frac{\alpha-\beta}{\epsilon}\right) U_{\tau 3} U_{e 3}^{*} \\
\widetilde{U}_{\tau 3} \widetilde{U}_{e 3}^{*} \simeq(1+\beta) U_{\tau 3} U_{e 3}^{*}-\alpha \beta \sin ^{2} \theta_{13} U_{\tau 1} U_{e 1}^{*}
\end{array}\right.
$$


and

$$
\widetilde{\triangle}_{\tau}:\left\{\begin{array}{l}
\widetilde{U}_{e 1} \widetilde{U}_{\mu 1}^{*} \simeq \frac{\alpha}{\epsilon} U_{e 1} U_{\mu 1}^{*}-\frac{1}{2}\left(1+\beta-\frac{\alpha+\beta}{\epsilon}\right) U_{e 3} U_{\mu 3}^{*}, \\
\widetilde{U}_{e 2} \widetilde{U}_{\mu 2}^{*} \simeq \frac{\alpha}{\epsilon} U_{e 2} U_{\mu 2}^{*}-\frac{1}{2}\left(1+\beta-\frac{\alpha-\beta}{\epsilon}\right) U_{e 3} U_{\mu 3}^{*} \\
\widetilde{U}_{e 3} \widetilde{U}_{\mu 3}^{*} \simeq(1+\beta) U_{e 3} U_{\mu 3}^{*}-\alpha \beta \sin ^{2} \theta_{13} U_{e 1} U_{\mu 1}^{*} .
\end{array}\right.
$$

One may check that the unitarity of each of the effective triangles holds up to the $\mathcal{O}(\alpha \beta)$, $\mathcal{O}\left(\sin ^{2} \theta_{13} \beta\right)$ or higher-order corrections in the above approximations. At this precision level the deviation of $\widetilde{\triangle}_{\mu}$ from $\triangle_{\mu}$ and the departure of $\widetilde{\triangle}_{\tau}$ from $\triangle_{\tau}$ are exactly the same, reflecting a kind of $\mu-\tau$ flavor symmetry between these two effective UTs [41]. Some further discussions about our approximate analytical results are in order.

(a) The $\widetilde{U}_{\alpha 3} \widetilde{U}_{\beta 3}^{*}$ side of the UT $\widetilde{\triangle}_{\gamma}$, where the subscripts $\alpha, \beta$ and $\gamma$ run cyclically over $e, \mu$ and $\tau$, is least sensitive to terrestrial matter effects. The reason is simply that $\widetilde{U}_{e 3}, \widetilde{U}_{\mu 3}$ and $\widetilde{U}_{\tau 3}$ only depend on the effective flavor mixing angles $\widetilde{\theta}_{13} \simeq \theta_{13}$ and $\widetilde{\theta}_{23} \simeq \theta_{23}$, which are almost insensitive to the matter-induced corrections when the neutrino beam energy $E$ is low. Hence $\widetilde{U}_{\alpha 3} \widetilde{U}_{\beta 3}^{*} \simeq U_{\alpha 3} U_{\beta 3}^{*}$ is a reasonably good approximation for $\widetilde{\triangle}_{\gamma}$ and $\triangle_{\gamma}$ in the low-energy region. In other words, the size and orientation of this side are essentially stable against terrestrial matter effects, and thus the deviation of $\widetilde{\triangle}_{\gamma}$ from $\triangle_{\gamma}$ is mainly attributed to the changes of the other two sides.

(b) The $\widetilde{U}_{\alpha 1} \widetilde{U}_{\beta 1}^{*}$ (or $\widetilde{U}_{\alpha 2} \widetilde{U}_{\beta 2}^{*}$ ) side of $\widetilde{\triangle}_{\gamma}$ consists of the corresponding $U_{\alpha 1} U_{\beta 1}^{*}\left(\right.$ or $\left.U_{\alpha 2} U_{\beta 2}^{*}\right)$ side of $\triangle_{\gamma}$ multiplied by a universal factor $\alpha / \epsilon$ and the $U_{\alpha 3} U_{\beta 3}^{*}$ side of $\triangle_{\gamma}$ multiplied by another factor. Because of

$$
\frac{|\alpha|}{\epsilon} \simeq \frac{1}{\sqrt{1-2 \cos 2 \theta_{12} \cos ^{2} \theta_{13} \frac{A}{\Delta_{21}}+\cos ^{4} \theta_{13}\left(\frac{A}{\Delta_{21}}\right)^{2}}},
$$

it becomes clear that this factor approaches 1 for $A \rightarrow 0$ and approximates to $1 /\left(2 \sin \theta_{12}\right) \simeq 0.91$ when $A \simeq \Delta_{21}$ holds (i.e., $\alpha \simeq \beta$ with $E \simeq 0.33 \mathrm{GeV}$ ) for a neutrino beam or to $1 /\left(2 \cos \theta_{12}\right) \simeq 0.60$ when $A \simeq \Delta_{21}$ holds for an antineutrino beam. In comparison, the term proportional to $U_{\alpha 3} U_{\beta 3}^{*}$ can change the orientation of the $\widetilde{U}_{\alpha 1} \widetilde{U}_{\beta 1}^{*}$ (or $\left.\widetilde{U}_{\alpha 2} \widetilde{U}_{\beta 2}^{*}\right)$ side, and its factors $[1-(\alpha \mp \beta) / \epsilon] / 2$ may appreciably deviate from zero even though $E$ is small. The deformation of the UT $\triangle_{\gamma}$ is therefore understandable.

(c) Note that the approximate analytical results in eqs. (3.8)-(3.11) are valid for the normal neutrino mass hierarchy. As for the inverted neutrino mass hierarchy with $\alpha<0$, the nine sides of the three effective Dirac UTs can be directly read off from eqs. (3.8)-(3.10) with the replacement $\epsilon \rightarrow-\epsilon$. In this case one may similarly discuss the deformation of each UT in the low-energy region for either a neutrino beam or an antineutrino beam. 
To be more explicit, let us look at the unique peak $\widetilde{\mathcal{J}}_{*} / \mathcal{J} \simeq|\alpha| / \epsilon_{*} \simeq 1 / \sin 2 \theta_{12}$ at the resonance point $\beta_{*} \simeq \alpha \cos 2 \theta_{12}$ in the leading-order approximation, as already discussed below eq. (2.17). In this special but interesting case the nine sides of the effective Dirac UTs can be simply expressed as follows: ${ }^{11}$

$\widetilde{\triangle}_{e}:\left\{\begin{array}{l}\widetilde{U}_{\mu 1} \widetilde{U}_{\tau 1}^{*} \simeq \frac{1}{\sin 2 \theta_{12}} U_{\mu 1} U_{\tau 1}^{*}-\frac{1-\tan \theta_{12}}{2} U_{\mu 3} U_{\tau 3}^{*} \simeq 1.09 U_{\mu 1} U_{\tau 1}^{*}-0.17 U_{\mu 3} U_{\tau 3}^{*}, \\ \widetilde{U}_{\mu 2} \widetilde{U}_{\tau 2}^{*} \simeq \frac{1}{\sin 2 \theta_{12}} U_{\mu 2} U_{\tau 2}^{*}-\frac{1-\cot \theta_{12}}{2} U_{\mu 3} U_{\tau 3}^{*} \simeq 1.09 U_{\mu 2} U_{\tau 2}^{*}+0.26 U_{\mu 3} U_{\tau 3}^{*}, \\ \widetilde{U}_{\mu 3} \widetilde{U}_{\tau 3}^{*} \simeq U_{\mu 3} U_{\tau 3}^{*} ;\end{array}\right.$

and

$$
\widetilde{\triangle}_{\mu}:\left\{\begin{array}{l}
\widetilde{U}_{\tau 1} \widetilde{U}_{e 1}^{*} \simeq \frac{1}{\sin 2 \theta_{12}} U_{\tau 1} U_{e 1}^{*}-\frac{1-\cot \theta_{12}}{2} U_{\tau 3} U_{e 3}^{*} \simeq 1.09 U_{\tau 1} U_{e 1}^{*}+0.26 U_{\tau 3} U_{e 3}^{*}, \\
\widetilde{U}_{\tau 2} \widetilde{U}_{e 2}^{*} \simeq \frac{1}{\sin 2 \theta_{12}} U_{\tau 2} U_{e 2}^{*}-\frac{1-\tan \theta_{12}}{2} U_{\tau 3} U_{e 3}^{*} \simeq 1.09 U_{\tau 2} U_{e 2}^{*}-0.17 U_{\tau 3} U_{e 3}^{*} \\
\widetilde{U}_{\tau 3} \widetilde{U}_{e 3}^{*} \simeq U_{\tau 3} U_{e 3}^{*} ;
\end{array}\right.
$$

and

$$
\widetilde{\triangle}_{\tau}:\left\{\begin{array}{l}
\widetilde{U}_{e 1} \widetilde{U}_{\mu 1}^{*} \simeq \frac{1}{\sin 2 \theta_{12}} U_{e 1} U_{\mu 1}^{*}-\frac{1-\cot \theta_{12}}{2} U_{e 3} U_{\mu 3}^{*} \simeq 1.09 U_{e 1} U_{\mu 1}^{*}+0.26 U_{e 3} U_{\mu 3}^{*} \\
\widetilde{U}_{e 2} \widetilde{U}_{\mu 2}^{*} \simeq \frac{1}{\sin 2 \theta_{12}} U_{e 2} U_{\mu 2}^{*}-\frac{1-\tan \theta_{12}}{2} U_{e 3} U_{\mu 3}^{*} \simeq 1.09 U_{e 2} U_{\mu 2}^{*}-0.17 U_{e 3} U_{\mu 3}^{*} \\
\widetilde{U}_{e 3} \widetilde{U}_{\mu 3}^{*} \simeq U_{e 3} U_{\mu 3}^{*},
\end{array}\right.
$$

where $\theta_{12} \simeq 33.48^{\circ}$ has been taken as a typical input value to illustrate the deviation of each effective UT from its fundamental counterpart in vacuum. In particular, the enhancement of $\widetilde{\mathcal{J}}$ and the deformation of each triangle become quite transparent. For example, eq. (3.14) leads us to the approximate relationship

$$
\widetilde{\mathcal{J}}_{*}=\operatorname{Im}\left(\widetilde{U}_{e 2} \widetilde{U}_{\mu 3} \widetilde{U}_{e 3}^{*} \widetilde{U}_{\mu 2}^{*}\right) \simeq \frac{1}{\sin 2 \theta_{12}} \operatorname{Im}\left(U_{e 2} U_{\mu 3} U_{e 3}^{*} U_{\mu 2}^{*}\right)=\frac{1}{\sin 2 \theta_{12}} \mathcal{J}
$$

at the resonance point $\beta_{*} \simeq \alpha \cos 2 \theta_{12}$ under discussion. In figure 4 we plot the three Dirac UTs in the complex plane by inputting the best-fit values of six neutrino oscillation parameters and taking the resonant beam energy $E_{*} \simeq 0.140 \mathrm{GeV}$ (or $0.123 \mathrm{GeV}$ ) for the normal (or inverted) neutrino mass ordering, corresponding to the peak of $\widetilde{\mathcal{J}} / \mathcal{J}$ shown in figure 2. Now the deformation of each UT becomes more intuitive, although the terrestrial matter effects in such a low-energy case are not very significant. Two comments are in order.

\footnotetext{
${ }^{11}$ In this case the matter-induced corrections to the three Dirac UTs are not very significant due to the smallness of $E_{*}$, but the corresponding analytical approximations are simple and instructive because they only involve a single known parameter $\theta_{12}$ at the leading-order level.
} 

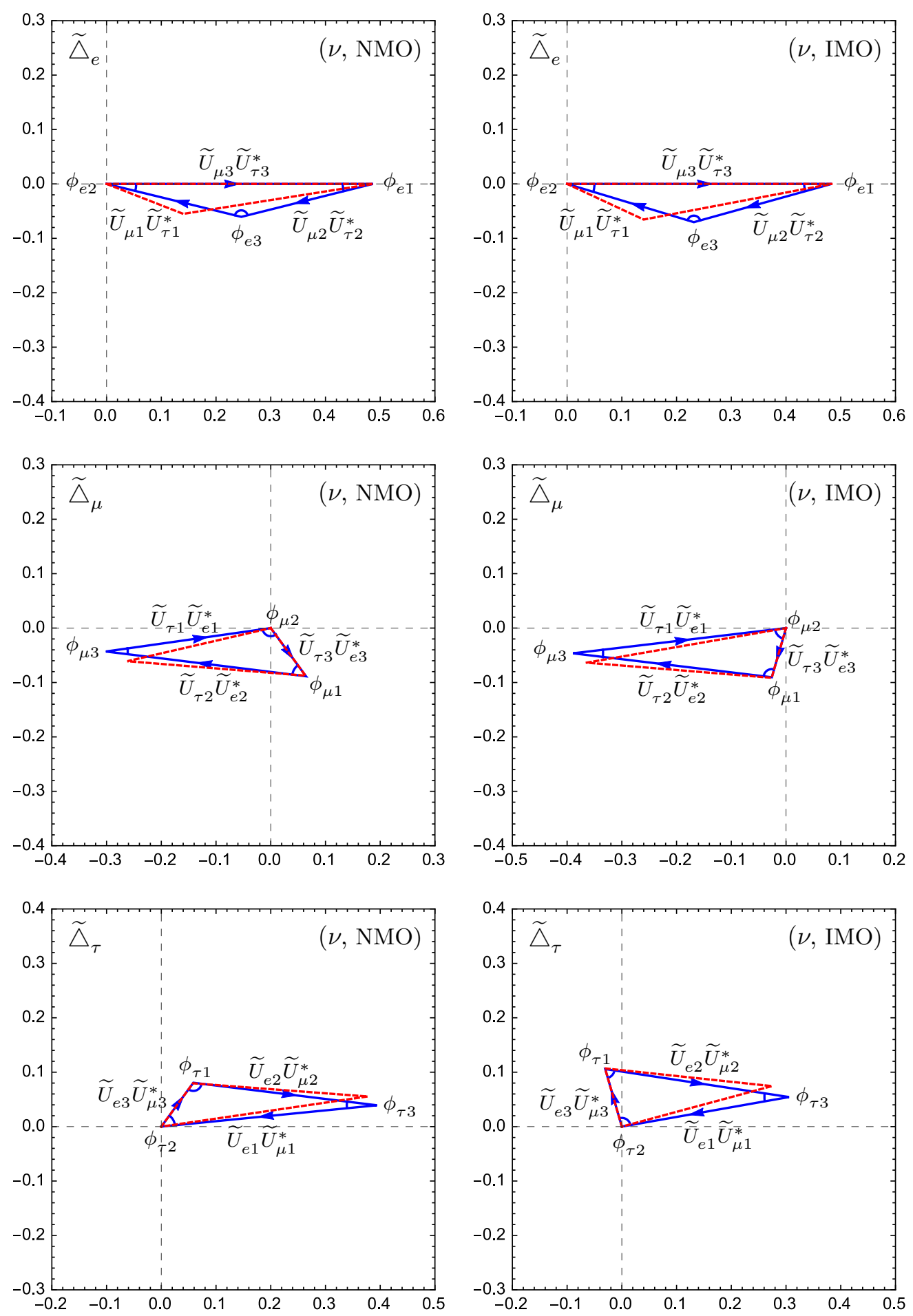

Figure 4. The matter-deformed Dirac UTs (blue and solid) as compared with their counterparts in vacuum (red and dashed), where the best-fit values of six neutrino oscillation parameters [4] have been input and the resonant beam energy $E_{*} \simeq 0.140 \mathrm{GeV}$ (or $0.123 \mathrm{GeV}$ ) has been taken for the normal (or inverted) neutrino mass ordering, corresponding to the peak of $\widetilde{\mathcal{J}} / \mathcal{J}$ shown in figure 2. 
(1) Figure 4 is a reflection of the real shapes of the fundamental and effective UTs based on the best-fit results of current neutrino oscillation data. The configurations of $\triangle_{\mu}$ (or $\triangle_{\tau}$ ) in the cases of normal and inverted mass neutrino hierarchies look quite different, simply because one of its three sides is proportional to $U_{e 3}^{*}=s_{13} e^{\mathrm{i} \delta}$ (or its complex conjugate) but the best-fit value of the CP-violating phase $\delta$ lies in two different quadrants in these two cases, as one can see in table 1. In comparison, the configuration of $\triangle_{e}$ is not so sensitive to the best-fit values of $\delta$ in the cases of normal and inverted mass hierarchies since its three sides do not directly depend on $U_{e 3}$ or equivalently $s_{13} e^{-\mathrm{i} \delta}$. As for the three effective Dirac UTs in matter, the same observations are true.

(2) Although the matter-induced corrections to the three fundamental UTs are not very significant, one can see a clear change in the orientations of two sides of each triangle at the resonance energy $E_{*}$. Our numerical results in figure 4 confirm the observations based on the analytical approximations made below eq. (3.10), implying that we have fully understood the matter-corrected behaviors of leptonic $\mathrm{CP}$ and $\mathrm{T}$ violation in the low-energy region.

To be more realistic, figures 5 and 6 show the matter-corrected Dirac UTs corresponding to the realistic accelerator-based $\mathrm{T} 2 \mathrm{~K}$ [5] and $\mathrm{NO} \nu \mathrm{A}[7]$ experiments which have the typical neutrino beam energies $0.6 \mathrm{GeV}$ and $2 \mathrm{GeV}$, respectively. In plotting these two figures we have input the best-fit values of six oscillation parameters and considered both the neutrino and antineutrino beams. Note that a description of antineutrino oscillations in matter actually involves $U^{*}$ and $-A$, but here we plot the relevant effective UTs defined in eq. (3.1) with $-A$ instead of their complex conjugate counterparts for an antineutrino beam so as to make a direct comparison between the same set of triangles in the neutrino (figure 5 with $A$ ) and antineutrino (figure 6 with $-A$ ) cases. $^{12}$ Some comments and discussions are in order.

(1) In both the $\mathrm{T} 2 \mathrm{~K}$ and $\mathrm{NO} \nu \mathrm{A}$ cases, the ratios of $\widetilde{\mathcal{J}}$ to $\mathcal{J}$ are strongly suppressed, as one can expect from figure 1 . To be specific, we obtain

$$
\begin{array}{r}
\mathrm{T} 2 \mathrm{~K}(E \simeq 0.6 \mathrm{GeV}): \frac{\mathcal{J}}{\mathcal{J}} \simeq \begin{cases}0.633 & \left(\nu \text { beam, } \Delta_{31}>0\right), \\
0.568 & \left(\nu \text { beam, } \Delta_{31}<0\right), \\
0.402 & \left(\bar{\nu} \text { beam, } \Delta_{31}>0\right), \\
0.448 & \left(\bar{\nu} \text { beam, } \Delta_{31}<0\right) ;\end{cases} \\
\mathrm{NO} \nu \mathrm{A}(E \simeq 2 \mathrm{GeV}): \frac{\mathcal{J}}{\mathcal{J}} \simeq \begin{cases}0.216 & \left(\nu \text { beam, } \Delta_{31}>0\right), \\
0.150 & \left(\nu \text { beam, } \Delta_{31}<0\right), \\
0.132 & \left(\bar{\nu} \text { beam, } \Delta_{31}>0\right), \\
0.190 & \left(\bar{\nu} \text { beam, } \Delta_{31}<0\right),\end{cases}
\end{array}
$$

\footnotetext{
${ }^{12}$ This point can be easily understood as follows. For example, $\widetilde{\triangle}_{e}$ is defined by the orthogonality relation $\widetilde{U}_{\mu 1} \widetilde{U}_{\tau 1}^{*}+\widetilde{U}_{\mu 2} \widetilde{U}_{\tau 2}^{*}+\widetilde{U}_{\mu 3} \widetilde{U}_{\tau 3}^{*}=0$ for a neutrino beam depending on $U$ and $A$. As for an antineutrino beam depending on $U^{*}$ and $-A$, the corresponding effective triangle is described by $\widetilde{U}_{\mu 1}^{*} \widetilde{U}_{\tau 1}+\widetilde{U}_{\mu 2}^{*} \widetilde{U}_{\tau 2}+\widetilde{U}_{\mu 3}^{*} \widetilde{U}_{\tau 3}=0$. What we have done in plotting figure 6 is simply to make a complex conjugation of this orthogonality relation, such that $\widetilde{\triangle}_{e}$ as a function of $A$ in figure 5 and $\widetilde{\triangle}_{e}$ as a function of $-A$ in figure 6 can be directly compared.
} 

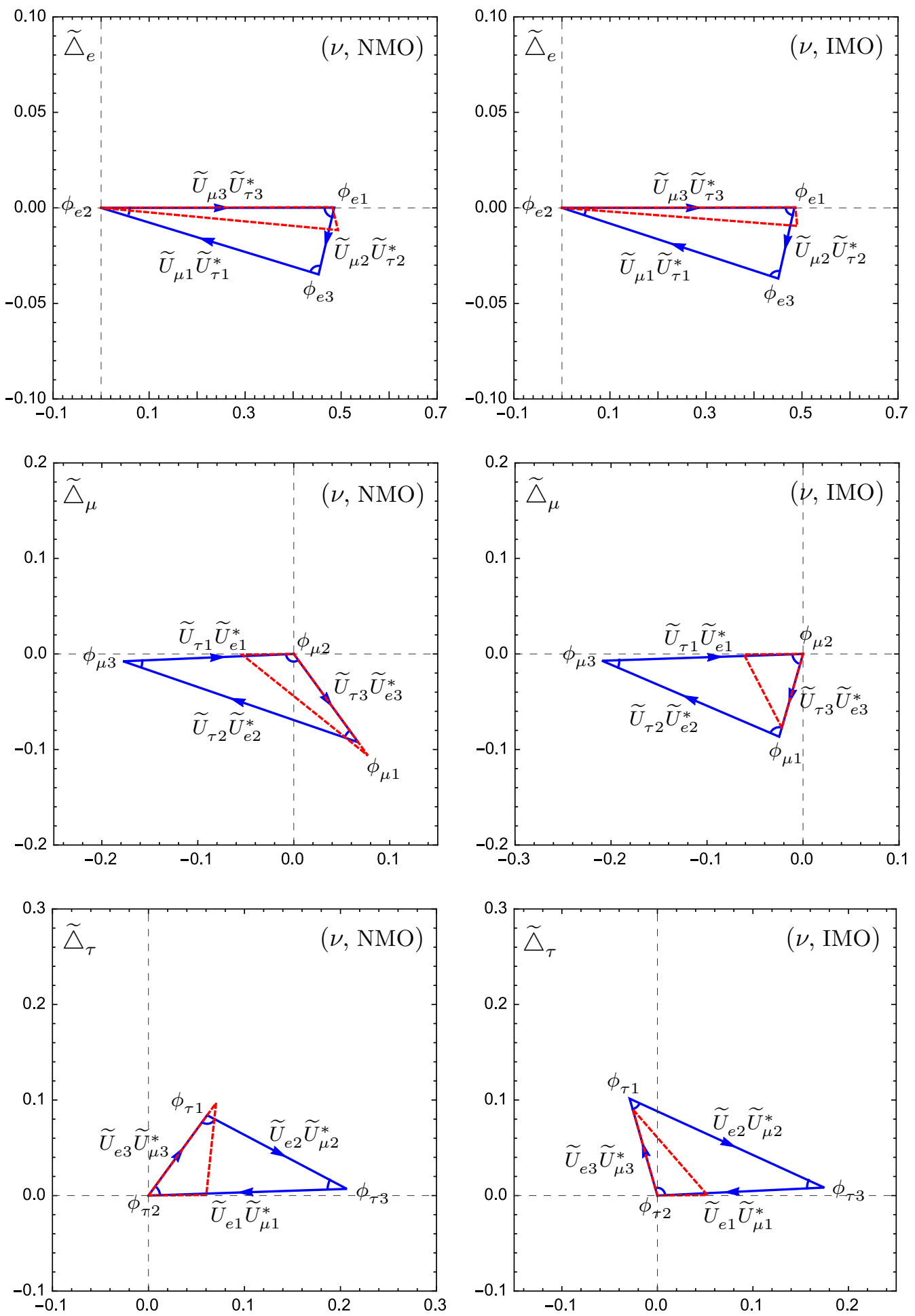

Figure 5. The real shapes of three matter-corrected Dirac UTs corresponding to the T2K (blue and solid, $E \simeq 0.6 \mathrm{GeV}$ ) and $\mathrm{NO} \nu \mathrm{A}$ (red and dashed, $E \simeq 2 \mathrm{GeV}$ ) neutrino oscillation experiments, where the best-fit values of six oscillation parameters [4] have been input. 

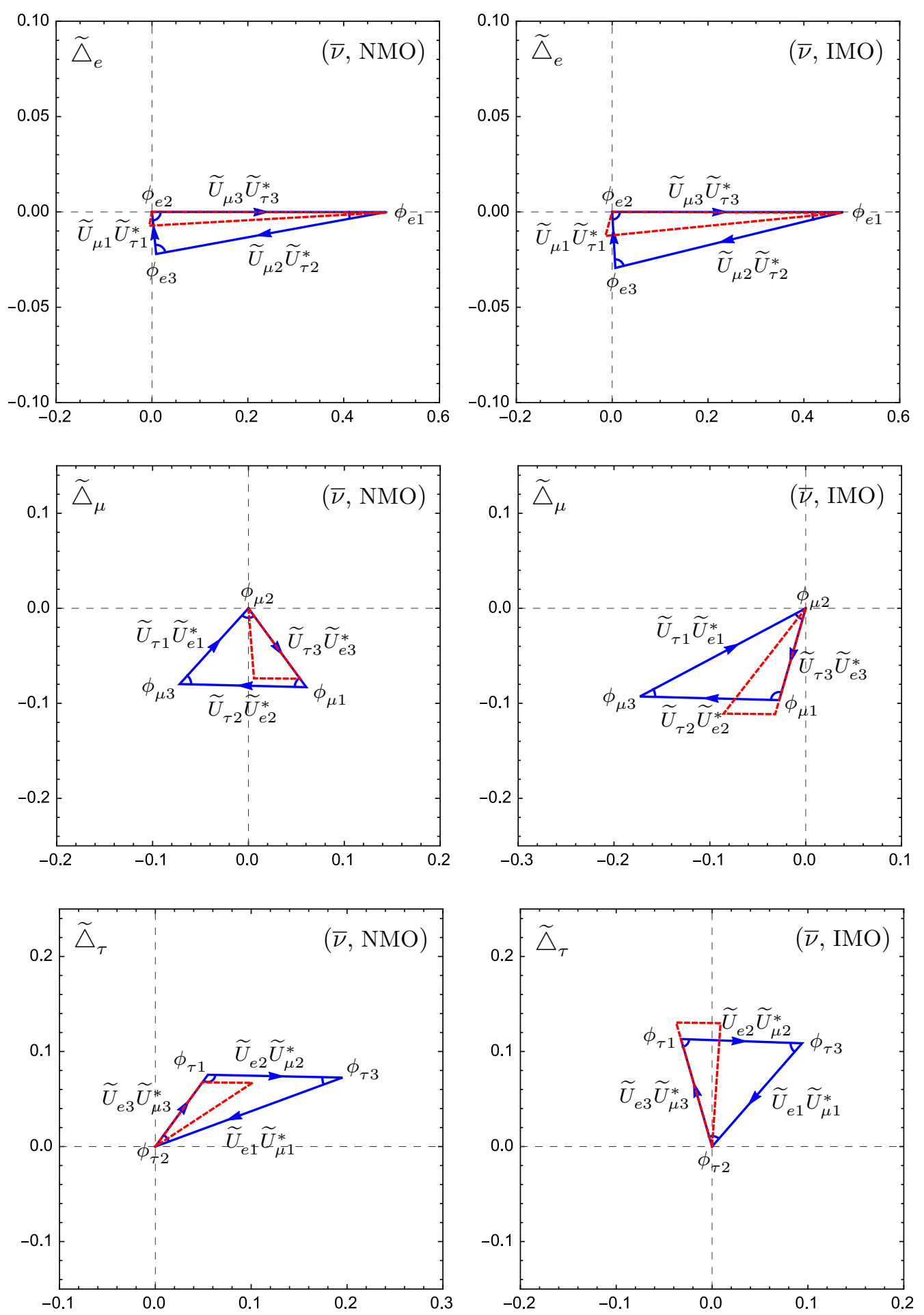

Figure 6. The real shapes of three matter-corrected Dirac UTs corresponding to the T2K (blue and solid, $E \simeq 0.6 \mathrm{GeV}$ ) and $\mathrm{NO} \nu \mathrm{A}$ (red and dashed, $E \simeq 2 \mathrm{GeV}$ ) antineutrino oscillation experiments, where the same inputs as those in figure 5 have been used. 
where $\mathcal{J} \simeq-0.0268$ (normal hierarchy) or -0.0316 (inverted hierarchy), calculated by inputting the best-fit values of $\theta_{12}, \theta_{13}, \theta_{23}$ and $\delta$ as listed in table 1 . Hence the areas of the UTs in figures 5 and 6 are much smaller than those in figure 4 , where $\widetilde{\mathcal{J}}_{*} / \mathcal{J} \simeq 1.10$ (normal hierarchy) or 1.07 (inverted hierarchy) for a neutrino beam with the same inputs.

(2) The $\widetilde{U}_{\alpha 3} \widetilde{U}_{\beta 3}^{*}$ side of $\widetilde{\triangle}_{\gamma}$, where the subscripts $\alpha, \beta$ and $\gamma$ run cyclically over $e, \mu$ and $\tau$, remains least sensitive to terrestrial matter effects as compared with the other two sides when the neutrino (or antineutrino) beam energy goes up. In either figure 5 or figure 6 , one may see the difference between the shapes or orientations of the same UT in the normal and inverted neutrino mass hierarchy cases. Such a difference mainly originates from the fact that the best-fit values of $\delta$ lie in two different quadrants in these two cases. When comparing one UT with respect to a neutrino beam with its counterpart with respect to an antineutrino beam (i.e., one UT in figure 5 and its counterpart in figure 6), we find that the changes associated with each triangle's shapes and orientations corresponding to $A \leftrightarrow-A$ are appreciable and even significant when $E$ increases - this effect is just the fake CP-violating asymmetry induced by terrestrial matter between $\nu_{\alpha} \rightarrow \nu_{\beta}$ and $\bar{\nu}_{\alpha} \rightarrow \bar{\nu}_{\beta}$ oscillations. The reason for this "asymmetry" is simply that the ordinary matter background is not symmetric under the CP transformation.

(3) The nine inner angles of the three effective Dirac UTs in matter can be defined as $\phi_{\alpha i} \equiv \arg \left[-\left(\widetilde{U}_{\beta j} \widetilde{U}_{\gamma j}^{*}\right) /\left(\widetilde{U}_{\beta k} \widetilde{U}_{\gamma k}^{*}\right)\right][44]$, where the Greek and Latin subscripts keep their cyclic running over $(e, \mu, \tau)$ and $(1,2,3)$, respectively. Taking the T2K and $\mathrm{NO} \nu \mathrm{A}$ experiments for example, we calculate these inner angles and list the numerical results in table 2, where the best-fit values of six neutrino oscillation parameters shown in table 1 have been input. It is obvious that terrestrial matter effects may significantly change the inner angles of the UTs, and therefore change their configurations and orientations.

\section{Neutrino oscillations and CP violation}

Now we turn to the possibility of measuring leptonic CP violation in neutrino oscillations in a low-energy or low-matter-density region. In practice the matter-corrected sides $\left|\widetilde{U}_{\alpha i} \widetilde{U}_{\beta i}^{*}\right|$ of three Dirac UTs and their corresponding Jarlskog parameter $\widetilde{\mathcal{J}}$ can be determined from a variety of long- or medium-baseline neutrino oscillation experiments [42]. The probabilities of $\nu_{\alpha} \rightarrow \nu_{\beta}$ oscillations in matter are given by

$$
\widetilde{P}\left(\nu_{\alpha} \rightarrow \nu_{\beta}\right)=\delta_{\alpha \beta}-4 \sum_{i<j} \operatorname{Re}\left(\widetilde{U}_{\alpha i} \widetilde{U}_{\beta j} \widetilde{U}_{\alpha j}^{*} \widetilde{U}_{\beta i}^{*}\right) \sin ^{2} \widetilde{F}_{j i}+8 \tilde{\mathcal{J}} \sum_{\gamma} \epsilon_{\alpha \beta \gamma} \prod_{i<j} \sin \widetilde{F}_{j i}
$$

where $\widetilde{F}_{j i} \equiv \widetilde{\Delta}_{j i} L /(4 E)$, and the Greek and Latin subscripts run over $(e, \mu, \tau)$ and $(1,2,3)$, respectively. Given the algebraic relationship

$$
\operatorname{Re}\left(\widetilde{U}_{\alpha i} \widetilde{U}_{\beta j} \widetilde{U}_{\alpha j}^{*} \widetilde{U}_{\beta i}^{*}\right)=\frac{1}{2}\left(\left|\widetilde{U}_{\alpha k} \widetilde{U}_{\beta k}^{*}\right|^{2}-\left|\widetilde{U}_{\alpha i} \widetilde{U}_{\beta i}^{*}\right|^{2}-\left|\widetilde{U}_{\alpha j} \widetilde{U}_{\beta j}^{*}\right|^{2}\right)
$$




\begin{tabular}{|c|c|c|c|c|c|c|}
\hline & \multicolumn{3}{|c|}{ Normal mass ordering (NMO) } & \multicolumn{3}{|c|}{ Inverted mass ordering (IMO) } \\
\hline & $\widetilde{U}=U$ & $E \simeq 0.6 \mathrm{GeV}$ & $E \simeq 2 \mathrm{GeV}$ & $\widetilde{U}=U$ & $E \simeq 0.6 \mathrm{GeV}$ & $E \simeq 2 \mathrm{GeV}$ \\
\hline \multirow{2}{*}{$\phi_{e 1}$} & \multirow{2}{*}{$9.0^{\circ}$} & $\left\{47.0^{\circ}(\nu)\right.$ & $\left\{134.8^{\circ}(\nu)\right.$ & \multirow{2}{*}{$10.8^{\circ}$} & $\left\{47.8^{\circ}(\nu)\right.$ & $\int 111.7^{\circ}(\nu)$ \\
\hline & & $2.6^{\circ}(\bar{\nu})$ & $0.8^{\circ}(\bar{\nu})$ & & $3.5^{\circ}(\bar{\nu})$ & $1.5^{\circ}(\bar{\nu})$ \\
\hline \multirow{2}{*}{$\phi_{e 2}$} & \multirow{2}{*}{$21.4^{\circ}$} & $\int 4.4^{\circ}(\nu)$ & $\int 1.4^{\circ}(\nu)$ & \multirow{2}{*}{$25.1^{\circ}$} & $\left\{4.7^{\circ}(\nu)\right.$ & $\left\{1.2^{\circ}(\nu)\right.$ \\
\hline & & $68.4^{\circ}(\bar{\nu})$ & $128.0^{\circ}(\bar{\nu})$ & & $77.4^{\circ}(\bar{\nu})$ & $138.3^{\circ}(\bar{\nu})$ \\
\hline \multirow{2}{*}{$\phi_{e 3}$} & \multirow{2}{*}{$149.6^{\circ}$} & $\int 128.6^{\circ}(\nu)$ & $\left\{43.8^{\circ}(\nu)\right.$ & \multirow{2}{*}{$144.1^{\circ}$} & $\int 127.5^{\circ}(\nu)$ & $\left\{67.1^{\circ}(\nu)\right.$ \\
\hline & & $109.0^{\circ}(\bar{\nu})$ & $51.2^{\circ}(\bar{\nu})$ & & $99.1^{\circ}(\bar{\nu})$ & $40.2^{\circ}(\bar{\nu})$ \\
\hline \multirow{2}{*}{$\phi_{\mu 1}$} & \multirow{2}{*}{$49.3^{\circ}$} & $\left\{34.9^{\circ}(\nu)\right.$ & $\left\{15.2^{\circ}(\nu)\right.$ & \multirow{2}{*}{$101.3^{\circ}$} & $\int 82.7^{\circ}(\nu)$ & $\int 43.2^{\circ}(\nu)$ \\
\hline & & $52.6^{\circ}(\bar{\nu})$ & $53.6^{\circ}(\bar{\nu})$ & & $104.5^{\circ}(\bar{\nu})$ & $105.3^{\circ}(\bar{\nu})$ \\
\hline \multirow{2}{*}{$\phi_{\mu 2}$} & \multirow{2}{*}{$112.9^{\circ}$} & $\int 123.5^{\circ}(\nu)$ & $\left\{125.2^{\circ}(\nu)\right.$ & \multirow{2}{*}{$64.1^{\circ}$} & $\int 72.0^{\circ}(\nu)$ & $\left\{73.5^{\circ}(\nu)\right.$ \\
\hline & & $78.0^{\circ}(\bar{\nu})$ & $31.5^{\circ}(\bar{\nu})$ & & $45.8^{\circ}(\bar{\nu})$ & $21.8^{\circ}(\bar{\nu})$ \\
\hline \multirow{2}{*}{$\phi_{\mu 3}$} & \multirow{2}{*}{$17.8^{\circ}$} & $\int 21.6^{\circ}(\nu)$ & $\left\{39.6^{\circ}(\nu)\right.$ & \multirow{2}{*}{$14.6^{\circ}$} & $\int 25.3^{\circ}(\nu)$ & $\int 63.3^{\circ}(\nu)$ \\
\hline & & $49.4^{\circ}(\bar{\nu})$ & $94.9^{\circ}(\bar{\nu})$ & & $29.7^{\circ}(\bar{\nu})$ & $52.9^{\circ}(\bar{\nu})$ \\
\hline \multirow{2}{*}{$\phi_{\tau 1}$} & \multirow{2}{*}{$121.7^{\circ}$} & $98.1^{\circ}(\nu)$ & $\left\{30.0^{\circ}(\nu)\right.$ & \multirow{2}{*}{$67.9^{\circ}$} & $49.5^{\circ}(\nu)$ & $\left\{25.1^{\circ}(\nu)\right.$ \\
\hline & & $124.8^{\circ}(\bar{\nu})$ & $125.6^{\circ}(\bar{\nu})$ & & $72.0^{\circ}(\bar{\nu})$ & $73.2^{\circ}(\bar{\nu})$ \\
\hline \multirow{2}{*}{$\phi_{\tau 2}$} & \multirow{2}{*}{$45.6^{\circ}$} & $\left\{52.1^{\circ}(\nu)\right.$ & $\left\{53.4^{\circ}(\nu)\right.$ & \multirow{2}{*}{$90.8^{\circ}$} & $\left\{103.3^{\circ}(\nu)\right.$ & $\left\{105.3^{\circ}(\nu)\right.$ \\
\hline & & $33.6^{\circ}(\bar{\nu})$ & $20.5^{\circ}(\bar{\nu})$ & & $56.8^{\circ}(\bar{\nu})$ & $19.9^{\circ}(\bar{\nu})$ \\
\hline \multirow{2}{*}{$\phi_{\tau 3}$} & \multirow{2}{*}{$12.7^{\circ}$} & $29.8^{\circ}(\nu)$ & $\left\{96.6^{\circ}(\nu)\right.$ & \multirow{2}{*}{$21.3^{\circ}$} & $\left\{27.2^{\circ}(\nu)\right.$ & $\left\{49.6^{\circ}(\nu)\right.$ \\
\hline & & $21.6^{\circ}(\bar{\nu})$ & $33.9^{\circ}(\bar{\nu})$ & & $51.2^{\circ}(\bar{\nu})$ & $86.9^{\circ}(\bar{\nu})$ \\
\hline
\end{tabular}

Table 2. A numerical illustration of terrestrial matter effects on the inner angles of the Dirac UTs in the T2K (with $E \simeq 0.6 \mathrm{GeV}$ ) and $\mathrm{NO} \nu \mathrm{A}$ (with $E \simeq 2 \mathrm{GeV}$ ) experiments, where the best-fit values of six neutrino oscillation parameters [4] have been typically input.

with $\alpha \neq \beta$ and $i \neq j \neq k$, one may then express the appearance $(\beta \neq \alpha)$ probabilities of neutrino oscillations in terms of the sides of the UTs and $\widetilde{\mathcal{J}}$ as follows:

$$
\begin{aligned}
\widetilde{P}\left(\nu_{\alpha} \rightarrow \nu_{\beta}\right)= & -2\left(\left|\widetilde{U}_{\alpha 3} \widetilde{U}_{\beta 3}^{*}\right|^{2}-\left|\widetilde{U}_{\alpha 1} \widetilde{U}_{\beta 1}^{*}\right|^{2}-\left|\widetilde{U}_{\alpha 2} \widetilde{U}_{\beta 2}^{*}\right|^{2}\right) \sin ^{2} \widetilde{F}_{21} \\
& -2\left(\left|\widetilde{U}_{\alpha 2} \widetilde{U}_{\beta 2}^{*}\right|^{2}-\left|\widetilde{U}_{\alpha 1} \widetilde{U}_{\beta 1}^{*}\right|^{2}-\left|\widetilde{U}_{\alpha 3} \widetilde{U}_{\beta 3}^{*}\right|^{2}\right) \sin ^{2} \widetilde{F}_{31} \\
& -2\left(\left|\widetilde{U}_{\alpha 1} \widetilde{U}_{\beta 1}^{*}\right|^{2}-\left|\widetilde{U}_{\alpha 2} \widetilde{U}_{\beta 2}^{*}\right|^{2}-\left|\widetilde{U}_{\alpha 3} \widetilde{U}_{\beta 3}^{*}\right|^{2}\right) \sin ^{2} \widetilde{F}_{32} \\
& +8 \widetilde{\mathcal{J}} \sum_{\gamma} \epsilon_{\alpha \beta \gamma} \sin \widetilde{F}_{21} \sin \widetilde{F}_{31} \sin \widetilde{F}_{32} .
\end{aligned}
$$

Of our particular interest are the $\nu_{\mu} \rightarrow \nu_{e}$ and $\bar{\nu}_{\mu} \rightarrow \bar{\nu}_{e}$ oscillations to probe leptonic $\mathrm{CP}$ violation. In this case it is the Dirac UT $\widetilde{\triangle}_{\tau}$ that fully determines the oscillation 
probabilities. Namely,

$$
\begin{aligned}
\widetilde{P}\left(\nu_{\mu} \rightarrow \nu_{e}\right)= & -2\left(\left|\widetilde{U}_{e 3} \widetilde{U}_{\mu 3}^{*}\right|^{2}-\left|\widetilde{U}_{e 1} \widetilde{U}_{\mu 1}^{*}\right|^{2}-\left|\widetilde{U}_{e 2} \widetilde{U}_{\mu 2}^{*}\right|^{2}\right) \sin ^{2} \widetilde{F}_{21} \\
& -2\left(\left|\widetilde{U}_{e 2} \widetilde{U}_{\mu 2}^{*}\right|^{2}-\left|\widetilde{U}_{e 1} \widetilde{U}_{\mu 1}^{*}\right|^{2}-\left|\widetilde{U}_{e 3} \widetilde{U}_{\mu 3}^{*}\right|^{2}\right) \sin ^{2} \widetilde{F}_{31} \\
& -2\left(\left|\widetilde{U}_{e 1} \widetilde{U}_{\mu 1}^{*}\right|^{2}-\left|\widetilde{U}_{e 2} \widetilde{U}_{\mu 2}^{*}\right|^{2}-\left|\widetilde{U}_{e 3} \widetilde{U}_{\mu 3}^{*}\right|^{2}\right) \sin ^{2} \widetilde{F}_{32} \\
& -8 \widetilde{\mathcal{J}} \sin \widetilde{F}_{21} \sin \widetilde{F}_{31} \sin \widetilde{F}_{32},
\end{aligned}
$$

and the corresponding expression of $\widetilde{P}\left(\bar{\nu}_{\mu} \rightarrow \bar{\nu}_{e}\right)$ can be directly read off from eq. (4.4) with the replacements $\mathcal{J} \rightarrow-\mathcal{J}$ and $A \rightarrow-A$.

To see an interplay between the fundamental physics and terrestrial matter effects in the probability of $\nu_{\mu} \rightarrow \nu_{e}$ oscillations in a more transparent way, let us make an analytical approximation for the expression of $\widetilde{P}\left(\nu_{\mu} \rightarrow \nu_{e}\right)$ in eq. (4.4), whose CP-conserving part is only related to the sides of $\widetilde{\triangle}_{\tau}$. Instead of adopting eq. (3.10), here we start from eq. (3.4) and make a higher-order analytical approximation to ensure a sufficient accuracy associated with $\widetilde{P}\left(\nu_{\mu} \rightarrow \nu_{e}\right)$ itself. That is,

$$
\begin{aligned}
& \widetilde{U}_{e 1} \widetilde{U}_{\mu 1}^{*}=\frac{\alpha}{\epsilon} U_{e 1} U_{\mu 1}^{*}+\frac{\alpha-\epsilon+\beta \cos ^{2} \theta_{13}-\epsilon \beta-\alpha \beta \cos 2 \theta_{12}+\beta^{2}}{2 \epsilon} U_{e 3} U_{\mu 3}^{*}, \\
& \widetilde{U}_{e 2} \widetilde{U}_{\mu 2}^{*}=\frac{\alpha}{\epsilon} U_{e 2} U_{\mu 2}^{*}+\frac{\alpha-\epsilon-\beta \cos ^{2} \theta_{13}-\epsilon \beta+\alpha \beta \cos 2 \theta_{12}-\beta^{2}}{2 \epsilon} U_{e 3} U_{\mu 3}^{*}, \\
& \widetilde{U}_{e 3} \widetilde{U}_{\mu 3}^{*}=(1+\beta) U_{e 3} U_{\mu 3}^{*} .
\end{aligned}
$$

With the help of eqs. (2.13), (2.16) and (4.5), we finally arrive at the result

$$
\begin{aligned}
\widetilde{P}\left(\nu_{\mu} \rightarrow\right. & \left.\nu_{e}\right) \simeq \alpha^{2} \sin ^{2} 2 \theta_{12} \cos ^{2} \theta_{13}\left(\cos ^{2} \theta_{23}-\sin ^{2} \theta_{13} \sin ^{2} \theta_{23}\right) \frac{\sin ^{2}\left(\epsilon F_{31}\right)}{\epsilon^{2}} \\
& +\frac{1}{2}(1+2 \beta) \sin ^{2} 2 \theta_{13} \sin ^{2} \theta_{23}\left[1-\cos \left(F_{*}-\beta F_{31}\right) \cos \left(\epsilon F_{31}\right)\right] \\
& +\frac{1}{2}(1+2 \beta)\left(\alpha \cos 2 \theta_{12}-\beta \cos ^{2} \theta_{13}\right) \sin ^{2} 2 \theta_{13} \sin ^{2} \theta_{23} \sin \left(F_{*}-\beta F_{31}\right) \frac{\sin \left(\epsilon F_{31}\right)}{\epsilon} \\
& +4 \mathcal{J} \alpha(1+\beta)\left(\alpha \cos 2 \theta_{12}-\beta \cos ^{2} \theta_{13}\right) \cot \delta \frac{\sin ^{2}\left(\epsilon F_{31}\right)}{\epsilon^{2}} \\
& -4 \mathcal{J} \alpha(1+\beta) \cos \left(\epsilon F_{31}\right) \frac{\sin \left(\epsilon F_{31}\right)}{\epsilon} \\
& +4 \frac{\mathcal{J}}{\sin \delta} \alpha(1+\beta) \sin \left(F_{*}-\beta F_{31}+\delta\right) \frac{\sin \left(\epsilon F_{31}\right)}{\epsilon}
\end{aligned}
$$

where $F_{*} \equiv \Delta_{*} L /(4 E)$ with $\Delta_{*} \equiv \Delta_{31}+\Delta_{32}$. Since the sign of $\Delta_{*}$ is always the same as that of $\Delta_{31}$ or $\Delta_{32}$, it can serve as a discriminator of the neutrino mass ordering in a medium-baseline reactor antineutrino oscillation experiment [45]. Of course, eq. (4.6) is valid for the normal neutrino mass ordering case. When the inverted mass hierarchy (i.e., $\left.\Delta_{31}<0\right)$ is concerned, the corresponding result can be easily obtained from eq. (4.6) with the replacement $\epsilon \rightarrow-\epsilon$, leading us to an expression which is formally the same as eq. (4.6). As for an antineutrino beam, the expression of $\widetilde{P}\left(\bar{\nu}_{\mu} \rightarrow \bar{\nu}_{e}\right)$ in the normal hierarchy case 

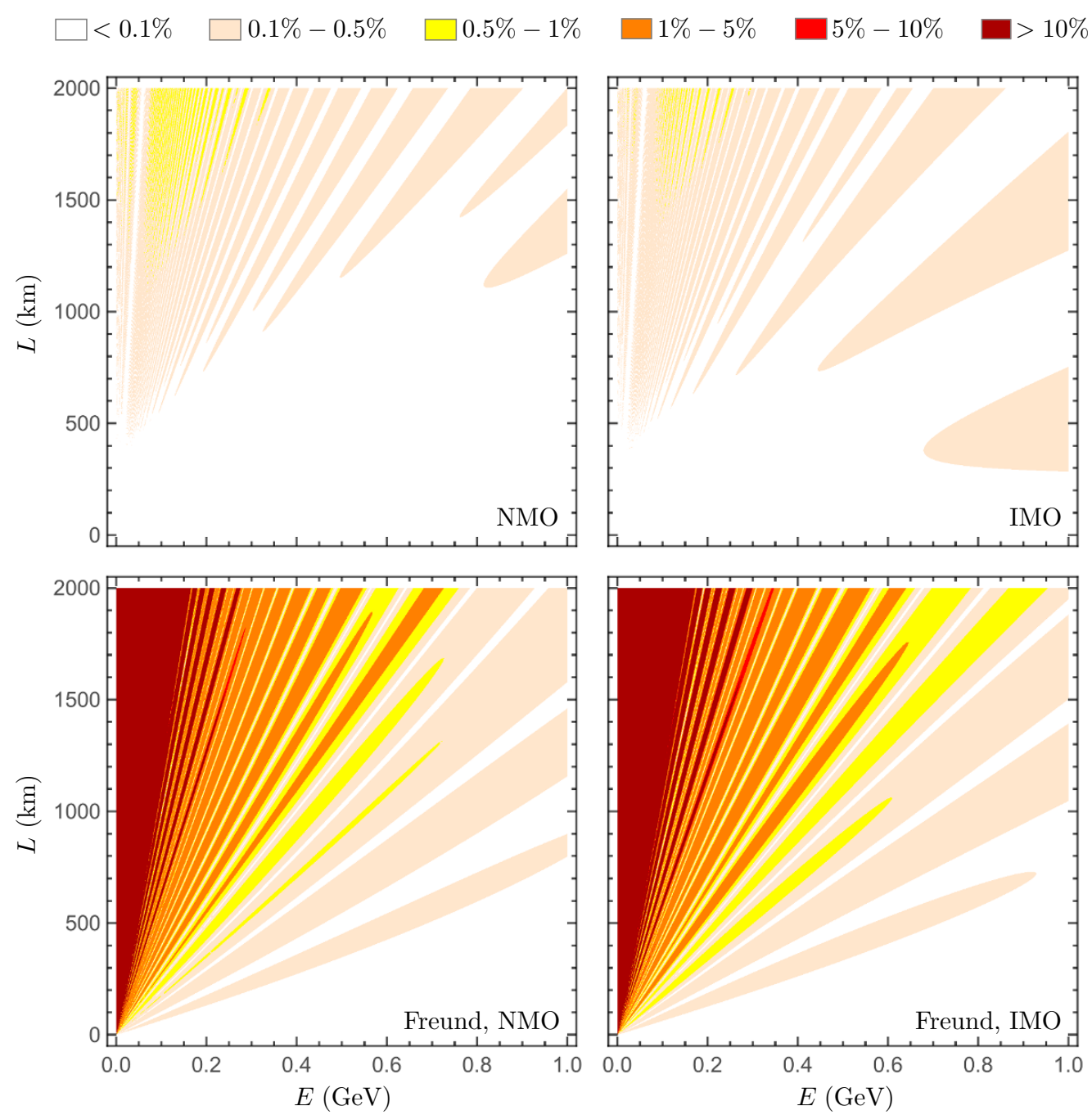

Figure 7. A comparison between the accuracies of our analytical approximations in eq. (4.6) and Freund's in ref. [15] by requiring $\delta \widetilde{P}\left(\nu_{\mu} \rightarrow \nu_{e}\right)$ defined in eq. (4.7) to be smaller than $0.1 \%$, $0.1 \%-0.5 \%, \cdots$. Here the best-fit values of relevant oscillation parameters [4], together with $A \simeq 2.28 \times 10^{-4} \mathrm{eV}^{2}(E / \mathrm{GeV})$, have been typically input.

can be directly read off from eq. (4.6) with the replacements $\delta \rightarrow-\delta$ and $A \rightarrow-A$. Note that $A \rightarrow-A$ is equivalent to $\beta \rightarrow-\beta$, implying a consequent change of $\epsilon$.

Different from Freund's analytical approximations for $\widetilde{P}\left(\nu_{\mu} \rightarrow \nu_{e}\right)$ [15], which mainly work in the $E \gtrsim 0.5 \mathrm{GeV}$ region, ours in eq. (4.6) can simply reproduce the corresponding vacuum result in the $A \rightarrow 0$ limit (i.e., in the absence of terrestrial matter effects). Although $\mathrm{Xu}$ has shown that Freund's result can be extended to cover the solar neutrino resonance region, it is expected to be numerically less accurate than our result. To verify this point, we illustrate the allowed parameter space of $E$ and $L$ for a given departure of the analyticalapproximation-based numerical result of $\widetilde{P}\left(\nu_{\mu} \rightarrow \nu_{e}\right)$ from the exact numerical result in figure 7 , in which $A \simeq 2.28 \times 10^{-4} \mathrm{eV}^{2}(E / \mathrm{GeV})$ is typically taken and the best-fit values 
of relevant oscillation parameters [4] are input. Namely, we require

$$
\delta \widetilde{P}\left(\nu_{\mu} \rightarrow \nu_{e}\right) \equiv\left|\widetilde{P}\left(\nu_{\mu} \rightarrow \nu_{e}\right)_{\text {exact }}-\widetilde{P}\left(\nu_{\mu} \rightarrow \nu_{e}\right)_{\text {approximate }}\right| \lesssim 0.1 \%, 0.1 \%-0.5 \%, \cdots,
$$

to see how small or how big the corresponding space of $E$ and $L$ is. Figure 7 clearly shows that our analytical approximations in eq. (4.6) are numerically more accurate than Freund's in the $E \lesssim 1 \mathrm{GeV}$ region, especially when $E$ is smaller and smaller.

Now let us compare between the numerical results of Freund's and ours in another way, by considering one proposed experiment (MOMENT with $L=150 \mathrm{~km}$ [13]) and two real ones (T2K with $L=295 \mathrm{~km}[5]$ and $\mathrm{NO} \nu \mathrm{A}$ with $L=810 \mathrm{~km} \mathrm{[7]).} \mathrm{Since} \mathrm{the} E<0.1 \mathrm{GeV}$ region is essentially irrelevant to these three experiments, we have restricted ourselves to the $E \gtrsim 0.1 \mathrm{GeV}$ region in our calculations. Figures 8 and 9 illustrate the behaviors of $\widetilde{P}\left(\nu_{\mu} \rightarrow\right.$ $\left.\nu_{e}\right)$ and $\delta \widetilde{P}\left(\nu_{\mu} \rightarrow \nu_{e}\right)$ for the normal and inverted neutrino mass hierarchies, respectively. We see that both Freund's analytical approximations and ours are actually good enough to describe the behaviors of matter-corrected $\nu_{\mu} \rightarrow \nu_{e}$ oscillations for the MOMENT and T2K experiments, although the accuracy of our approximations is certainly much better. In contrast, Freund's result is much better than ours for the $\mathrm{NO} \nu \mathrm{A}$ experiment, simply because the latter involves $E \gtrsim 1 \mathrm{GeV}$. In short, our new approximations provide an alternative analytical way for understanding the matter-modified behaviors of $\nu_{\mu} \rightarrow \nu_{e}$ and $\bar{\nu}_{\mu} \rightarrow \bar{\nu}_{e}$ oscillations in the $0.1 \mathrm{GeV} \lesssim E \lesssim 1 \mathrm{GeV}$ region.

In the following we focus on a low-energy medium-baseline neutrino oscillation experiment which is capable of probing leptonic CP-violating asymmetry

$$
\widetilde{\mathcal{A}}_{\mathrm{CP}} \equiv \widetilde{\mathcal{A}}_{\mathcal{J}}+\widetilde{\mathcal{A}}_{\mathrm{F}} \equiv \widetilde{P}\left(\nu_{\mu} \rightarrow \nu_{e}\right)-\widetilde{P}\left(\bar{\nu}_{\mu} \rightarrow \bar{\nu}_{e}\right)
$$

in which $\widetilde{\mathcal{A}}_{\mathcal{J}}$ stands for the genuine CP-violating effect governed by the nontrivial value of Dirac phase $\delta,{ }^{13}$ and $\widetilde{\mathcal{A}}_{\mathrm{F}}$ denotes the fake asymmetry arising from an asymmetry between terrestrial matter and antimatter. The latter must disappear when the "matter" parameter $A$ is switched off. With the help of eq. (4.6) and its counterpart for $\widetilde{P}\left(\bar{\nu}_{\mu} \rightarrow \bar{\nu}_{e}\right)$, one may obtain the simplified expressions of $\widetilde{\mathcal{A}}_{\mathcal{J}}$ and $\widetilde{\mathcal{A}}_{\mathrm{F}}$ as

$$
\widetilde{\mathcal{A}}_{\mathcal{J}} \simeq-16 \mathcal{J} F_{21} \sin ^{2} F_{31} \simeq \mathcal{A}_{\mathcal{J}} \equiv-16 \mathcal{J} \sin F_{21} \sin F_{31} \sin F_{32},
$$

and

$$
\begin{aligned}
\widetilde{\mathcal{A}}_{\mathrm{F}} \simeq 2 \beta\{ & \sin ^{2} 2 \theta_{13} \sin ^{2} \theta_{23}\left[2 \sin ^{2} F_{31}-(1+\alpha) F_{31} \sin \left(2 F_{31}\right)+\alpha \sin ^{2} \theta_{12} F_{31}^{2} \cos \left(2 F_{31}\right)\right] \\
& \left.-8 \alpha \mathcal{J} \cot \delta F_{31}^{2} \cos ^{2} F_{31}\right\},
\end{aligned}
$$

if $\sin \left(\epsilon F_{31}\right) \simeq \epsilon F_{31}$ holds as a reasonable approximation. In this case it becomes transparent that the fake $\mathrm{CP}$-violating asymmetry $\widetilde{\mathcal{A}}_{\mathrm{F}}$ is proportional to the matter parameter $A$, while the genuine CP-violating asymmetry $\widetilde{\mathcal{A}}_{\mathcal{J}}$ in matter is essentially equal to its counterpart in

\footnotetext{
${ }^{13}$ Note that $\widetilde{\mathcal{A}}_{\mathcal{J}}$ as a CP-violating asymmetry is associated with both matter $(A)$ and antimatter $(-A)$, while $\widetilde{\mathcal{A}}_{\mathrm{T}}$ defined in eq. (1.2) is the T-violating asymmetry and thus depends only on matter.
} 

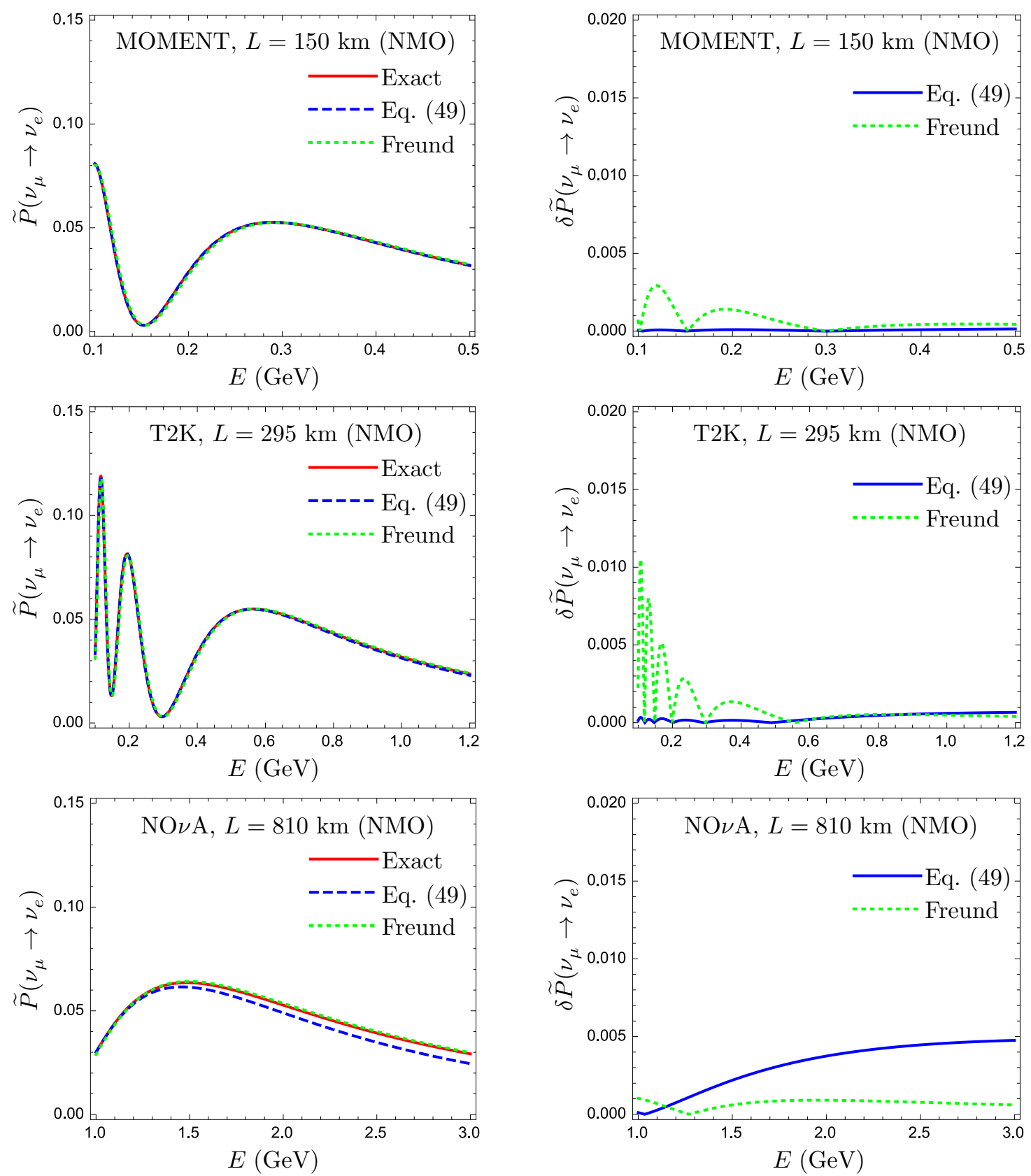

Figure 8. A comparison between the numerical accuracies of our analytical approximations in eq. (4.6) and Freund's in ref. [15] for the MOMENT, T2K and NO $\nu \mathrm{A}$ experiments in the normal neutrino mass ordering case. Here the best-fit values of relevant oscillation parameters [4], together with $A \simeq 2.28 \times 10^{-4} \mathrm{eV}^{2}(E / \mathrm{GeV})$, have been typically input. 

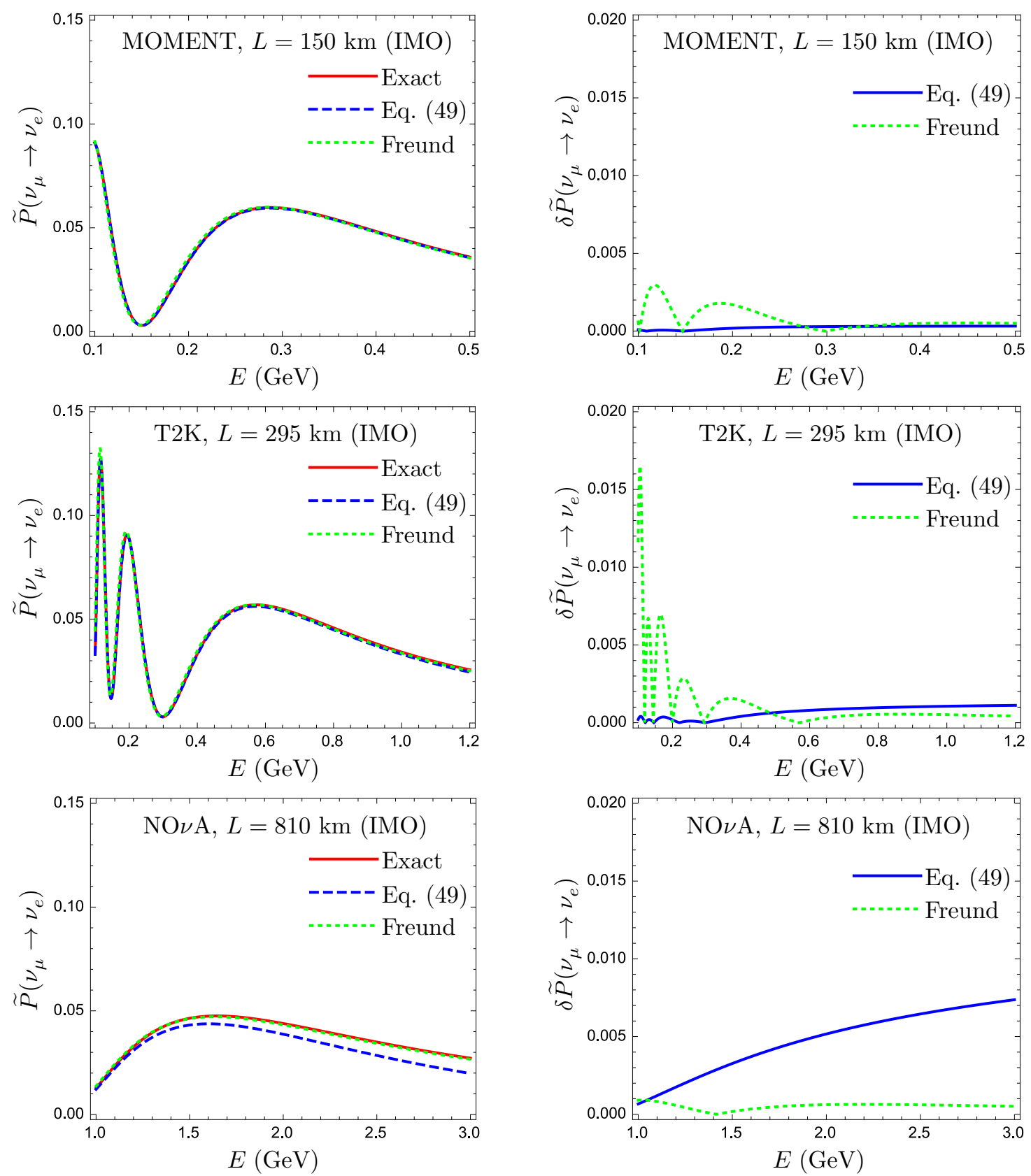

Figure 9. A comparison between the numerical accuracies of our analytical approximations in eq. (4.6) and Freund's in ref. [15] for the MOMENT, T2K and NO $\nu \mathrm{A}$ experiments in the inverted neutrino mass ordering case. Here the best-fit values of relevant oscillation parameters [4], together with $A \simeq 2.28 \times 10^{-4} \mathrm{eV}^{2}(E / \mathrm{GeV})$, have been typically input. 

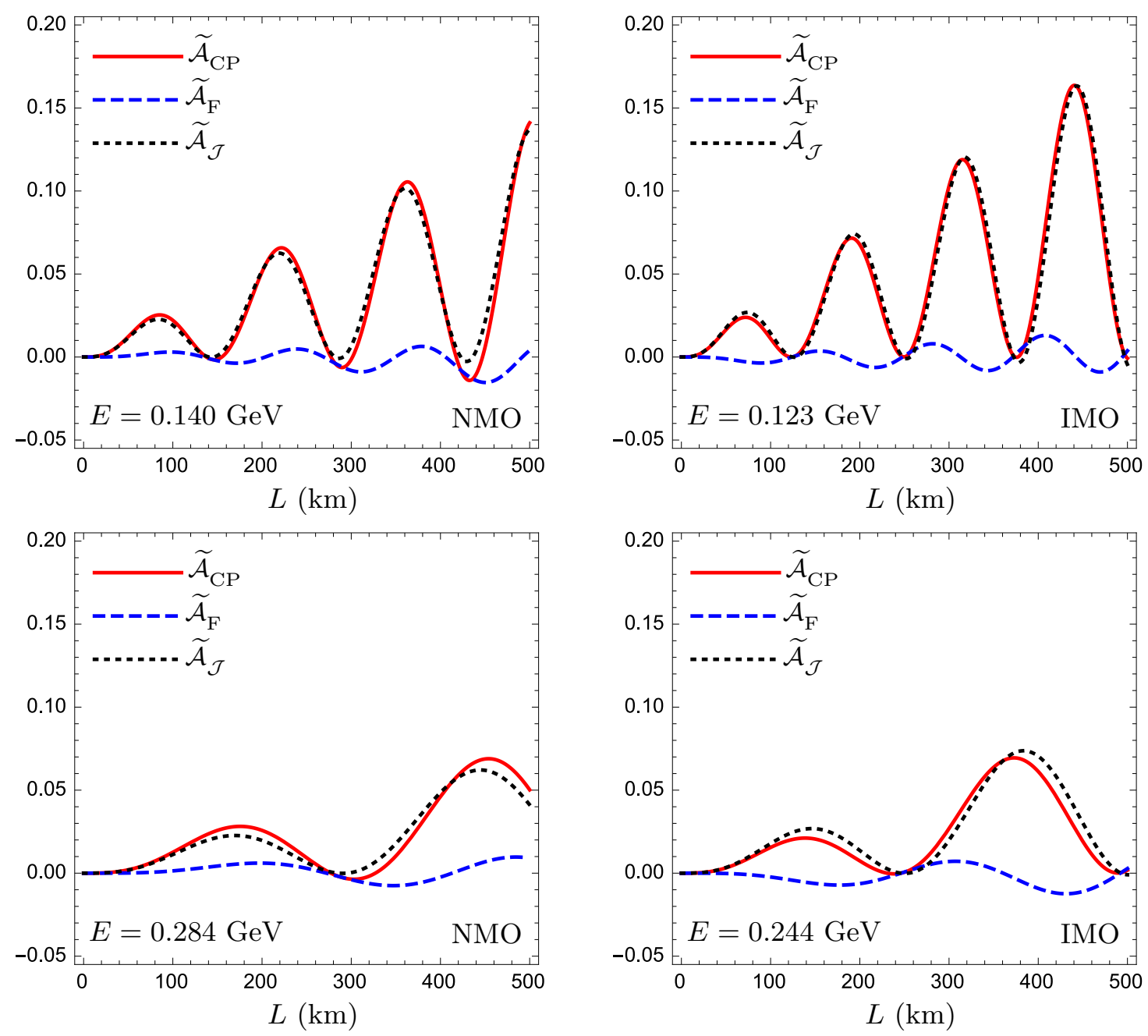

Figure 10. The CP-violating asymmetry $\widetilde{\mathcal{A}}_{\mathrm{CP}}$ and its genuine $\left(\widetilde{\mathcal{A}}_{\mathcal{J}}\right)$ and fake $\left(\widetilde{\mathcal{A}}_{\mathrm{F}}\right)$ components for $\nu_{\mu} \rightarrow \nu_{e}$ and $\bar{\nu}_{\mu} \rightarrow \bar{\nu}_{e}$ oscillations in matter, where the benchmark beam energies $E=0.140 \mathrm{GeV}$ (or $0.123 \mathrm{GeV}$ ) and $E=0.284 \mathrm{GeV}$ (or $0.244 \mathrm{GeV}$ ) are taken for the normal (inverted) mass ordering, and the best-fit values of neutrino oscillation parameters [4] have been input.

vacuum. In fact, the result in eq. (4.9) is well known $[24,28,43],{ }^{14}$ but the one in eq. (4.10) is new and instructive.

To illustrate the above observations in a numerical way, let us take two benchmark values of the neutrino beam energy $E$ and plot the asymmetries $\widetilde{\mathcal{A}}_{\mathcal{J}}, \widetilde{\mathcal{A}}_{\mathrm{F}}$ and $\widetilde{\mathcal{A}}_{\mathrm{CP}}=$ $\widetilde{\mathcal{A}}_{\mathcal{J}}+\widetilde{\mathcal{A}}_{\mathrm{F}}$ as functions of the baseline length $L$ in figure 10 , where the best-fit values of six neutrino oscillation parameters have been input. These two benchmark beam energies are just $E_{*}$ and $E_{0} \simeq 2 E_{*}$, corresponding to the $\widetilde{\mathcal{J}}_{*} / \mathcal{J}$ peak and the nontrivial $\widetilde{\mathcal{J}} / \mathcal{J}=1$ point as pointed out in section 2 . One can see that $\widetilde{\mathcal{A}}_{\mathrm{CP}} \simeq \widetilde{\mathcal{A}}_{\mathcal{J}}$ is an acceptable approximation in the $E \simeq E_{*}$ case, and the deviation of $\widetilde{\mathcal{A}}_{\mathrm{CP}}$ from $\widetilde{\mathcal{A}}_{\mathcal{J}}$ can be appreciable when $L$ becomes

\footnotetext{
${ }^{14}$ For example, it has been shown that the equality $\widetilde{\mathcal{J}} \sin \widetilde{F}_{21} \sin \widetilde{F}_{31} \sin \widetilde{F}_{32} \simeq \mathcal{J} \sin F_{21} \sin F_{31} \sin F_{32}$ holds to a good degree of accuracy provided the neutrino beam energy $E$ and the baseline length $L$ satisfy the condition $10^{-7}(L / \mathrm{km})^{2}(\mathrm{GeV} / E) \ll 1[43]$.
} 


\begin{tabular}{|c|ccccc|}
\hline & & \multicolumn{2}{c}{ Normal mass ordering } & \multicolumn{2}{c|}{ Inverted mass ordering } \\
\hline \multirow{5}{*}{ 1st peak } & & \multicolumn{2}{|c}{$E(\mathrm{GeV})$} & \multicolumn{2}{c|}{$E(\mathrm{GeV})$} \\
& & 0.140 & 0.284 & 0.123 & 0.244 \\
\hline \multirow{3}{*}{ 2nd peak } & $L(\mathrm{~km})$ & 85.20 & 175.5 & 71.90 & 138.5 \\
& $\widetilde{\mathcal{A}}_{\mathrm{CP}}$ & 0.025 & 0.028 & 0.024 & 0.021 \\
& $\widetilde{\mathcal{A}}_{\mathcal{J}} / \widetilde{\mathcal{A}}_{\mathrm{CP}}$ & 0.893 & 0.801 & 1.121 & 1.256 \\
& $L(\mathrm{~km})$ & 221.7 & 454.0 & 190.7 & 372.9 \\
& $\widetilde{\mathcal{A}}_{\mathrm{CP}}$ & 0.066 & 0.069 & 0.072 & 0.069 \\
& $\widetilde{\mathcal{A}}_{\mathcal{J}} / \widetilde{\mathcal{A}}_{\mathrm{CP}}$ & 0.948 & 0.892 & 1.031 & 1.043 \\
\hline
\end{tabular}

Table 3. The benchmark values of $E, L, \widetilde{\mathcal{A}}_{\mathrm{CP}}$ and $\widetilde{\mathcal{A}}_{\mathcal{J}} / \widetilde{\mathcal{A}}_{\mathrm{CP}}$ associated with the first and second peaks of the CP-violating asymmetry $\widetilde{\mathcal{A}}_{\mathrm{CP}}$ shown in figure 10 .

larger simply because the matter-induced fake asymmetry $\widetilde{\mathcal{A}}_{\mathrm{F}}$ increases with $L$ as implied in eq. (4.10). Although it is possible to obtain much larger CP-violating asymmetries when the baseline length $L$ is properly large, a price to pay for the growth of $L$ is the decrease of the neutrino flux luminosity because the latter is proportional to $L^{-2}$ [28]. For this reason, we focus on the first two peaks of $\widetilde{\mathcal{A}}_{\mathrm{CP}}$ in figure 10 . The values of $E, L, \widetilde{\mathcal{A}}_{\mathrm{CP}}$ and $\widetilde{\mathcal{A}}_{\mathcal{J}} / \widetilde{\mathcal{A}}_{\mathrm{CP}}$ associated with these two peaks are summarized in table 3 . Two comments are in order.

(a) In the case of a normal neutrino mass hierarchy, $\widetilde{\mathcal{A}}_{\mathcal{J}} / \widetilde{\mathcal{A}}_{\mathrm{CP}}<1$ holds on the peaks, implying that the fake $\mathrm{CP}$-violating asymmetry $\widetilde{\mathcal{A}}_{\mathrm{F}}$ contributes in a positive way. In contrast, the contribution of $\widetilde{\mathcal{A}}_{\mathrm{F}}$ is negative for the inverted neutrino mass ordering, and hence $\widetilde{\mathcal{A}}_{\mathcal{J}} / \widetilde{\mathcal{A}}_{\mathrm{CP}}>1$ holds in this case.

(b) Given $E \simeq E_{*}$ for the first peak of $\widetilde{\mathcal{A}}_{\mathrm{CP}}$, the corresponding baseline length $L$ is about $85.20 \mathrm{~km}$ (or $71.90 \mathrm{~km}$ ) in the $\Delta_{31}>0$ (or $\Delta_{31}<0$ ) case. When $E \simeq 2 E_{*}$ is taken, the value of $L$ is roughly doubled. The situation is similar for the second peak of $\widetilde{\mathcal{A}}_{\mathrm{CP}}$. Of course, a realistic experiment should optimize both $E$ and $L$ to make $\widetilde{\mathcal{A}}_{\mathrm{CP}}$ easily observable.

Furthermore, we plot the effective probabilities $\widetilde{P}\left(\nu_{\mu} \rightarrow \nu_{e}\right)$ and $\widetilde{P}\left(\bar{\nu}_{\mu} \rightarrow \bar{\nu}_{e}\right)$ changing with the baseline length $L$ in figure 11, where the inputs are exactly the same as those used for plotting figure 10. Since these two probabilities depend on $\pm A$ respectively, they receive different contributions from terrestrial matter effects and thus their peaks correspond to different values of $L$. The difference between $\widetilde{P}\left(\nu_{\mu} \rightarrow \nu_{e}\right)$ and $\widetilde{P}\left(\bar{\nu}_{\mu} \rightarrow \bar{\nu}_{e}\right)$ is just the CPviolating asymmetry $\widetilde{\mathcal{A}}_{\mathrm{CP}}$ as illustrated in figure 10 . Note that $\widetilde{\mathcal{A}}_{\mathrm{CP}}$ is essentially insensitive to the neutrino mass hierarchy in the leading-order approximation, because it is dominated by the $\widetilde{\mathcal{A}}_{\mathcal{J}}$ term which is insensitive to the sign of $\Delta_{31}$. This observation implies that a reasonable determination of the $\mathrm{CP}$-violating effect in the lepton sector (or equivalently, the size of $\delta$ ) should in principle be possible in such a low-energy medium-baseline neutrino oscillation experiment even before the sign of $\Delta_{31}$ is measured. 

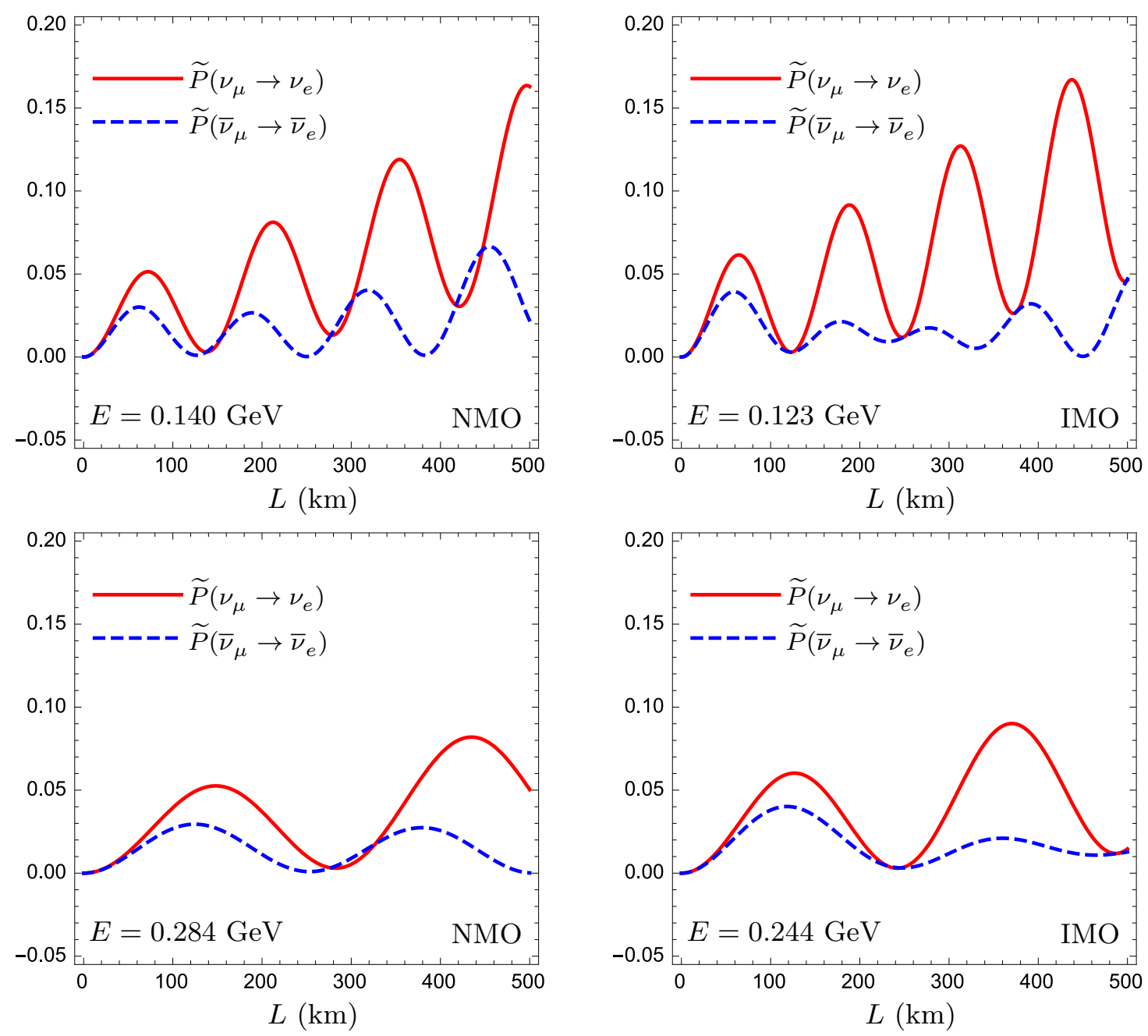

Figure 11. The probabilities of $\nu_{\mu} \rightarrow \nu_{e}$ and $\bar{\nu}_{\mu} \rightarrow \bar{\nu}_{e}$ oscillations in matter, where the benchmark beam energies $E=0.140 \mathrm{GeV}$ (or $0.123 \mathrm{GeV}$ ) and $E=0.284 \mathrm{GeV}$ (or $0.244 \mathrm{GeV}$ ) are taken for the normal (inverted) mass ordering, and the best-fit values of neutrino oscillation parameters [4] have been input.

We hope that some of our results obtained in this work will be helpful for the design of a low-energy oscillation experiment to explore leptonic CP violation. The proposed MOMENT project [13] is just an experiment of this type. The neutrino flux of the MOMENT is expected to peak in the $0.15 \mathrm{GeV} \lesssim E \lesssim 0.20 \mathrm{GeV}$ region, which happens to coincide with the $E_{*} \lesssim E \lesssim 2 E_{*}$ region recommended above. In other words, this experiment is capable of probing the effects of CP violation in $\nu_{\mu} \rightarrow \nu_{e}$ and $\bar{\nu}_{\mu} \rightarrow \bar{\nu}_{e}$ oscillations with little matter-induced suppression. The present studies indicate that the optimal baseline length of the MOMENT experiment should be around $L \simeq 150 \mathrm{~km}[13,46]$, which is also within the expectation shown in figures 10 and 11. But the bottlenecks to the physics reach of this experiment include how to achieve a sufficiently intense neutrino (or antineutrino) flux and how to achieve a sufficiently high suppression of the atmospheric neutrino background, as pointed out and discussed in depth by Blennow et al. in ref. [46]. In this connection 
we plan to go into details of the feasibility and physics potential of the MOMENT project elsewhere in collaboration with its team members [47].

In addition to the MOMENT facility, the $\mathrm{ESS} \nu \mathrm{SB}$ project - a very intense neutrino super-beam for the measurement of leptonic CP violation - has recently been proposed based on the European Spallation Source Linac [14]. Its neutrino beam energy and baseline length are expected to lie in the $0.2 \mathrm{GeV} \lesssim E \lesssim 0.5 \mathrm{GeV}$ range and the $300 \mathrm{~km} \lesssim L \lesssim$ $600 \mathrm{~km}$ range, respectively. It is obvious that the lower-energy and shorter-baseline part of this parameter space is consistent with our recommendation about $E$ and $L$ made above. In fact, our analytical approximations are valid for the whole space of $E$ and $L$ of the $\operatorname{ESS} \nu \mathrm{SB}$ experiment, and hence they will be very helpful to understand the numerical analysis of this experiment's sensitivity to CP violation and matter contamination [48].

We stress that a low-energy medium-baseline neutrino oscillation experiment can not only help probe leptonic $\mathrm{CP}$ violation but also help test the other properties of lepton flavor mixing. Therefore, a further study of this possibility is desirable [47].

\section{Summary}

We have developed a new set of analytical approximations for the probabilities of $\nu_{\mu} \rightarrow \nu_{e}$ and $\bar{\nu}_{\mu} \rightarrow \bar{\nu}_{e}$ oscillations in matter to understand the effects of leptonic CP violation in a possible low-energy medium-baseline experiment with the beam energy $E \lesssim 1 \mathrm{GeV}$. Our primary motivation comes from the fact that the previous works of this kind, such as the popular one done by Freund [15], are subject to the $E \gtrsim 1 \mathrm{GeV}$ (or $E \gtrsim 0.5 \mathrm{GeV}$ ) region for a long-baseline oscillation experiment. We have shown that our analytical approximations are numerically more accurate than those made by Freund in the $E \lesssim 1 \mathrm{GeV}$ region, and thus they are expected to be particularly applicable for the MOMENT, ESS $\nu \mathrm{SM}$ and T2K experiments. The new analytical approximations can also help us to easily understand why the matter-corrected Jarlskog parameter $\widetilde{\mathcal{J}}$ peaks at the resonance energy $E_{*} \simeq 0.14 \mathrm{GeV}$ (or $0.12 \mathrm{GeV}$ ) for the normal (or inverted) neutrino mass hierarchy, and how the three Dirac unitarity triangles are deformed due to the terrestrial matter contamination. Finally we have affirmed that a medium-baseline neutrino oscillation experiment with the beam energy $E$ lying in the $E_{*} \lesssim E \lesssim 2 E_{*}$ range is capable of exploring leptonic $\mathrm{CP}$ violation with little matter-induced suppression.

Of course, more detailed works have to be done to combine our analytical results with a given experiment, such as the MOMENT project, by considering both the neutrino beam issues and the detector issues. We plan to focus on such important but complicated issues elsewhere in collaboration with the MOMENT team [47].

\section{Acknowledgments}

We are indebted to Yu-Feng Li and Shun Zhou for their useful discussions and comments. One of us (Z.Z.X.) is also grateful to Fumihiro Takayama for his warm hospitality during the Chinese New Year at the Yukawa Institute for Theoretical Physics of Kyoto University, 
where part of this work was done. The present research is supported in part by the National Natural Science Foundation of China under grant No. 11135009.

Open Access. This article is distributed under the terms of the Creative Commons Attribution License (CC-BY 4.0), which permits any use, distribution and reproduction in any medium, provided the original author(s) and source are credited.

\section{References}

[1] Particle Data Group collaboration, K.A. Olive et al., Review of particle physics, Chin. Phys. C 38 (2014) 090001 [InSPIRE].

[2] F. Capozzi, G.L. Fogli, E. Lisi, A. Marrone, D. Montanino and A. Palazzo, Status of three-neutrino oscillation parameters, circa 2013, Phys. Rev. D 89 (2014) 093018 [arXiv: 1312.2878] [INSPIRE].

[3] D.V. Forero, M. Tortola and J.W.F. Valle, Neutrino oscillations refitted, Phys. Rev. D 90 (2014) 093006 [arXiv: 1405.7540] [INSPIRE].

[4] M.C. Gonzalez-Garcia, M. Maltoni and T. Schwetz, Updated fit to three neutrino mixing: status of leptonic CP-violation, JHEP 11 (2014) 052 [arXiv:1409.5439] [INSPIRE].

[5] T2K collaboration, K. Abe et al., Measurement of neutrino oscillation parameters from muon neutrino disappearance with an off-axis beam, Phys. Rev. Lett. 111 (2013) 211803 [arXiv: 1308.0465] [INSPIRE].

[6] T2K collaboration, K. Abe et al., Precise measurement of the neutrino mixing parameter $\theta_{23}$ from muon neutrino disappearance in an off-axis beam, Phys. Rev. Lett. 112 (2014) 181801 [arXiv: 1403.1532] [INSPIRE].

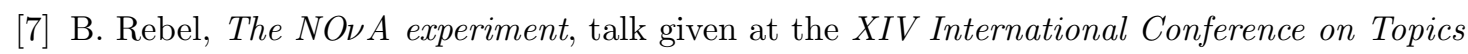
in Astroparticle and Underground Physics (TAUP 2015), September 7-15, Torino, Italy (2015).

[8] DAYA BAY collaboration, F.P. An et al., Spectral measurement of electron antineutrino oscillation amplitude and frequency at Daya Bay, Phys. Rev. Lett. 112 (2014) 061801 [arXiv: 1310.6732] [INSPIRE].

[9] DaYa BAY collaboration, F.P. An et al., New Measurement of Antineutrino Oscillation with the Full Detector Configuration at Daya Bay, Phys. Rev. Lett. 115 (2015) 111802 [arXiv: 1505.03456] [INSPIRE].

[10] P. Shanahan, Long-baseline and atmospheric neutrino experiments, talk given at the 27th International Symposium on Lepton Photon Interactions at High Energies, August 17-22, Ljubljana, Slovenia (2015).

[11] F. Capozzi, E. Lisi, A. Marrone, D. Montanino and A. Palazzo, Neutrino masses and mixings: status of known and unknown $3 \nu$ parameters, Nucl. Phys. B 908 (2016) 218 [arXiv: 1601.07777] [INSPIRE].

[12] Y. Wang and Z.-z. Xing, Neutrino masses and flavor oscillations, arXiv:1504.06155 [INSPIRE].

[13] J. Cao et al., Muon-decay medium-baseline neutrino beam facility, Phys. Rev. ST Accel. Beams 17 (2014) 090101 [arXiv:1401.8125] [INSPIRE]. 
[14] ESSnuSB collaboration, E. Baussan et al., A very intense neutrino super beam experiment for leptonic CP-violation discovery based on the European spallation source linac, Nucl. Phys. B 885 (2014) 127 [arXiv: 1309.7022] [INSPIRE].

[15] M. Freund, Analytic approximations for three neutrino oscillation parameters and probabilities in matter, Phys. Rev. D 64 (2001) 053003 [hep-ph/0103300] [INSPIRE].

[16] X.-J. Xu, Why is the neutrino oscillation formula expanded in $\Delta m_{21}^{2} / \Delta m_{31}^{2}$ still accurate near the solar resonance in matter?, JHEP 10 (2015) 090 [arXiv:1502.02503] [INSPIRE].

[17] B. Pontecorvo, Mesonium and anti-mesonium, Sov. Phys. JETP 6 (1957) 429 [Zh. Eksp. Teor. Fiz. 33 (1957) 549] [INSPIRE].

[18] Z. Maki, M. Nakagawa and S. Sakata, Remarks on the unified model of elementary particles, Prog. Theor. Phys. 28 (1962) 870 [InSPIRE].

[19] B. Pontecorvo, Neutrino experiments and the problem of conservation of leptonic charge, Sov. Phys. JETP 26 (1968) 984 [Zh. Eksp. Teor. Fiz. 53 (1967) 1717] [InSPIRE].

[20] C. Jarlskog, Commutator of the quark mass matrices in the standard electroweak model and a measure of maximal CP-violation, Phys. Rev. Lett. 55 (1985) 1039 [INSPIRE].

[21] L. Wolfenstein, Neutrino oscillations in matter, Phys. Rev. D 17 (1978) 2369 [INSPIRE].

[22] S.P. Mikheev and A. Yu. Smirnov, Resonance amplification of oscillations in matter and spectroscopy of solar neutrinos, Sov. J. Nucl. Phys. 42 (1985) 913 [Yad. Fiz. 42 (1985) 1441] [INSPIRE].

[23] P.I. Krastev and S.T. Petcov, Resonance amplification and $t$ violation effects in three neutrino oscillations in the Earth, Phys. Lett. B 205 (1988) 84 [INSPIRE].

[24] E.K. Akhmedov, P. Huber, M. Lindner and T. Ohlsson, T violation in neutrino oscillations in matter, Nucl. Phys. B 608 (2001) 394 [hep-ph/0105029] [INSPIRE].

[25] V.A. Naumov, Three neutrino oscillations in matter, CP-violation and topological phases, Int. J. Mod. Phys. D 1 (1992) 379 [inSPIRE].

[26] P.F. Harrison and W.G. Scott, $C P$ and T violation in neutrino oscillations and invariance of Jarlskog's determinant to matter effects, Phys. Lett. B 476 (2000) 349 [hep-ph/9912435] [INSPIRE].

[27] Z.-z. Xing, Sum rules of neutrino masses and CP-violation in the four neutrino mixing scheme, Phys. Rev. D 64 (2001) 033005 [hep-ph/0102021] [INSPIRE].

[28] H. Minakata and H. Nunokawa, Measuring leptonic CP-violation by low-energy neutrino oscillation experiments, Phys. Lett. B 495 (2000) 369 [hep-ph/0004114] [INSPIRE].

[29] H. Fritzsch and Z.-z. Xing, Mass and flavor mixing schemes of quarks and leptons, Prog. Part. Nucl. Phys. 45 (2000) 1 [hep-ph/9912358] [INSPIRE].

[30] J.A. Aguilar-Saavedra and G.C. Branco, Unitarity triangles and geometrical description of CP-violation with Majorana neutrinos, Phys. Rev. D 62 (2000) 096009 [hep-ph/0007025] [INSPIRE].

[31] Z.-z. Xing and J.-y. Zhu, Leptonic unitarity triangles and effective mass triangles of the Majorana neutrinos, Nucl. Phys. B 908 (2016) 302 [arXiv:1511.00450] [INSPIRE].

[32] S. Toshev, On T violation in matter neutrino oscillations, Mod. Phys. Lett. A 6 (1991) 455 [INSPIRE]. 
[33] V.D. Barger, K. Whisnant, S. Pakvasa and R.J.N. Phillips, Matter effects on three-neutrino oscillations, Phys. Rev. D 22 (1980) 2718 [INSPIRE].

[34] H.W. Zaglauer and K.H. Schwarzer, The mixing angles in matter for three generations of neutrinos and the MSW mechanism, Z. Phys. C 40 (1988) 273 [INSPIRE].

[35] Z.-z. Xing, New formulation of matter effects on neutrino mixing and CP-violation, Phys. Lett. B 487 (2000) 327 [hep-ph/0002246] [INSPIRE].

[36] A. Cervera et al., Golden measurements at a neutrino factory, Nucl. Phys. B 579 (2000) 17 [Erratum ibid. B 593 (2001) 731] [hep-ph/0002108] [INSPIRE].

[37] Y.-F. Li and S. Luo, Neutrino oscillation probabilities in matter with direct and indirect unitarity violation in the lepton mixing matrix, Phys. Rev. D 93 (2016) 033008 [arXiv: 1508.00052] [INSPIRE].

[38] I. Mocioiu and R. Shrock, Matter effects on neutrino oscillations in long baseline experiments, Phys. Rev. D 62 (2000) 053017 [hep-ph/0002149] [INSPIRE].

[39] Z.-z. Xing, Flavor mixing and CP-violation of massive neutrinos, Int. J. Mod. Phys. A 19 (2004) 1 [hep-ph/0307359] [INSPIRE].

[40] H. Zhang and Z.-z. Xing, Leptonic unitarity triangles in matter, Eur. Phys. J. C 41 (2005) 143 [hep-ph/0411183] [INSPIRE].

[41] Z.-z. Xing and Z.-h. Zhao, A review of $\mu-\tau$ flavor symmetry in neutrino physics, Rept. Prog. Phys. 79 (2016) 076201 [arXiv: 1512.04207] [INSPIRE].

[42] Z.-z. Xing and H. Zhang, Reconstruction of the neutrino mixing matrix and leptonic unitarity triangles from long-baseline neutrino oscillations, Phys. Lett. B 618 (2005) 131 [hep-ph/0503118] [INSPIRE].

[43] Z.-z. Xing, Leptonic commutators and clean T violation in neutrino oscillations, Phys. Rev. D 88 (2013) 017301 [arXiv:1304.7606] [INSPIRE].

[44] S. Luo, Dirac lepton angle matrix vs. Majorana lepton angle matrix and their renormalization group running behaviours, Phys. Rev. D 85 (2012) 013006 [arXiv:1109.4260] [INSPIRE].

[45] Y.-F. Li, Y. Wang and Z.-z. Xing, Terrestrial matter effects on reactor antineutrino oscillations at JUNO or RENO-50: how small is small?, arXiv:1605.00900 [INSPIRE].

[46] M. Blennow, P. Coloma and E. Fernández-Martinez, The MOMENT to search for CP-violation, JHEP 03 (2016) 197 [arXiv: 1511.02859] [INSPIRE].

[47] Y. F. Li and other members of the MOMENT experiment, private communications.

[48] T. Ohlsson, H. Zhang and S. Zhou, Probing the leptonic Dirac CP-violating phase in neutrino oscillation experiments, Phys. Rev. D 87 (2013) 053006 [arXiv: 1301.4333] [INSPIRE]. 